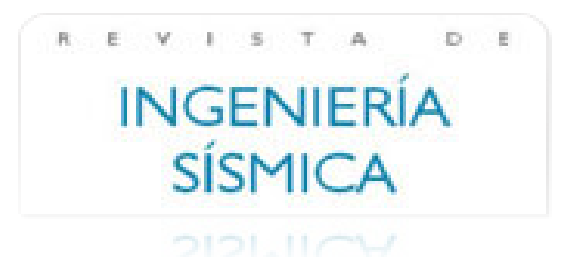

\section{Revista de Ingeniería Sísmica}

ISSN: 0185-092X

javiles@tlaloc.imta.mx

Sociedad Mexicana de Ingeniería Sísmica

México

Terán Gilmore, Amador; Virto Cambray, Neftalí

DISEÑO BASADO EN DESPLAZAMIENTOS DE EDIFICACIONES BAJAS RIGIDIZADAS CON

CONTRAVIENTOS DESADHERIDOS

Revista de Ingeniería Sísmica, núm. 80, enero-junio, 2009, pp. 1-29

Sociedad Mexicana de Ingeniería Sísmica

Distrito Federal, México

Disponible en: http://www.redalyc.org/articulo.oa?id=61812113002

Cómo citar el artículo

- Número completo

- Más información del artículo

- Página de la revista en redalyc.org

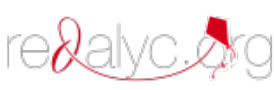

Sistema de Información Científica

Red de Revistas Científicas de América Latina, el Caribe, España y Portugal

Proyecto académico sin fines de lucro, desarrollado bajo la iniciativa de acceso abierto 


\title{
DISEÑO BASADO EN DESPLAZAMIENTOS DE EDIFICACIONES BAJAS RIGIDIZADAS CON CONTRAVIENTOS DESADHERIDOS
}

\author{
Amador Terán Gilmore ${ }^{(1)}$ y Neftalí Virto Cambray ${ }^{(2)}$
}

\begin{abstract}
RESUMEN
Se presenta una metodología basada en el control del desplazamiento lateral para el diseño por desempeño de un sistema de contravientos desadheridos y la estructura que los recibe. La metodología aplica al caso de estructuras de baja altura, cuyo comportamiento se caracteriza por no exhibir efectos importantes de flexión a nivel global. Se aplica la metodología propuesta para el diseño sísmico de una edificación de cinco pisos ubicada en la Zona del Lago del D.F. A partir de evaluar las características mecánicas globales de dicha edificación, y de evaluar el desempeño sísmico de la misma ante excitaciones sísmicas generadas en esa zona, se concluye que la metodología propuesta da lugar a un nivel adecuado de diseño sismorresistente.
\end{abstract}

\begin{abstract}
This paper introduces a displacement-based methodology for the performance-based design of a system of unbonded braces and the structure that receives it. The methodology applies to the case of low height structures, whose behavior is not significantly influenced by global flexural behavior. The methodology is applied to the seismic design of a five-story building located in the Lake Zone of Mexico City. From the evaluation of the global mechanical characteristics of the building and of its seismic performance when subjected to ground motions generated in that zone, it is concluded that the proposed methodology yields an adequate level of seismic design.
\end{abstract}

\section{INTRODUCCIÓN}

Al estudiar las razones por las cuales varios eventos sísmicos recientes han resultado en pérdidas económicas y humanas excesivas, la comunidad internacional de ingeniería sísmica ha empezado a entender con claridad que el nivel de daño estructural y no estructural en una estructura sismorresistente, así como el daño en su contenido, se deriva de la deformación y/o movimiento excesivo de la misma.

La innovación en ingeniería sísmica puede entenderse a partir del planteamiento de sistemas estructurales, ya sea tradicionales o innovadores, que puedan controlar adecuadamente el nivel de daño en las estructuras a través de controlar su deformación y movimiento durante las excitaciones sísmicas. Estudios recientes indican que las estructuras dúctiles desplantadas en la Zona del Lago del D. F.,

Artículo recibido el 7 de junio de 2007 y aprobado para su publicación el 11 de marzo de 2008. Se aceptarán comentarios y/o discusiones hasta cinco meses después de su publicación

1 Profesor, Universidad Autónoma Metropolitana, Departamento de Materiales, Av. San Pablo 180, Col. Reynosa, México 02200, D.F. tga@correo.azc.uam.mx

2 Gerente de Proyecto, Alonso y Asociados, Carretera Mexico-Toluca 1725, Despacho C-5, Col. Lomas de Palo Alto, México 05110, D.F. neftali@alonsoasociados.com.mx 
particularmente aquellas cuyo periodo dominante de vibración se acerca al periodo dominante del terreno, pueden verse sujetas a demandas severas de deformación plástica. Varios estudios analíticos muestran la preocupación de investigadores mexicanos alrededor de este tema (Terán 1998, Rodríguez y Ariztizabal 1999, Huerta y Reinoso 2002, Bojórquez y Ruiz 2004, Arroyo y Ordaz 2007, Terán y Bahena 2008, Terán y Espinoza 2008).

Se ha sugerido que una forma de proteger las estructuras del efecto de las demandas severas de deformación plástica acumulada consiste en diseñarlas para que acomoden durante la excitación sísmica demandas máximas de deformación que sean del orden del 50\% al 60\% de su deformación última (Bertero 1997, Panagiotakos y Fardis 2001). Dado que la resistencia lateral de una estructura sismorresistente es la propiedad mecánica que controla las demandas máximas y acumuladas de deformación plástica, el diseño contra fatiga de bajo número de ciclos en la Zona del Lago del D.F. ha dado lugar a niveles de diseño de resistencia que resultan relativamente altos. La aplicación de metodologías de diseño que consideren explícitamente las demandas acumuladas de deformación plástica ha dado lugar a estructuras muy robustas con un alto contenido de acero (Sánchez y Terán 2008, Terán y Simón 2008).

El nivel de diseño que se requiere para garantizar la seguridad estructural de las construcciones bajo estas circunstancias resulta en soluciones estructurales caras. Una forma de aliviar este problemática consiste en desarrollar sistemas de disipación pasiva de energía para las estructuras ubicadas en la Zona del Lago de Ciudad de México. Este trabajo representa el inicio de una serie de esfuerzos por desarrollar en México: A) Un sistema pasivo de disipación de energía basado en el uso de contravientos desadheridos, y B) Metodologías de diseño que hagan posible el uso racional de este sistema.

Se presenta una serie de estudios analíticos que exploran la pertinencia de utilizar contravientos desadheridos en edificios de baja altura ubicados en la Zona del Lago del D.F. Como parte de estos estudios se define el tipo de acero con el cual deben fabricarse los contravientos para obtener un buen balance entre el desempeño estructural de la edificación para los estados límite de servicio y seguridad. Además se desarrolla una metodología de diseño por desempeño (basada en el control del desplazamiento lateral) para el diseño sísmico del sistema de contravientos y la estructura que los recibe. Cabe mencionar que aunque el tratamiento que se le da en este artículo al sistema de contravientos desadheridos corresponde al diseño de una estructura nueva, los planteamientos pueden ser fácilmente adaptados para la rehabilitación de estructuras existentes. En cuanto a sus limitaciones, debe enfatizarse que la metodología propuesta solo debe utilizarse en edificaciones cuyo comportamiento global no esté influenciado de manera significativa por componentes de deformación global por flexión.

\section{CONTRAVIENTOS DESADHERIDOS}

Entre las razones por las cuales es conveniente añadir dispositivos disipadores de energía a las estructuras sismorresistentes, pueden mencionarse las siguientes: A) Disminuir las demandas de velocidad, aceleración y fuerza cortante de entrepiso; y B) Controlar las demandas plásticas en los elementos estructurales que bajan las cargas gravitacionales.

En términos generales, los sistemas de disipación de energía pueden clasificarse en:

- Pasivos. Las propiedades mecánicas de estos sistemas permanecen casi constantes durante la excitación sísmica. Ejemplos de estos sistemas son los aisladores de base con amortiguamiento adicional, dispositivos de disipación de energía viscosa y visco-elástica, y dispositivos de disipación de energía por fricción o fluencia del material. 
- Activos. Están constituidos por dispositivos que ajustan sus características mecánicas en función de la respuesta del edificio.

A corto plazo es realista desarrollar e implantar en México sistemas pasivos de disipación de energía basados en la fluencia del material (Badillo 2000, Virto 2006). Una condición que debe observar un dispositivo que disipa energía con base en fluencia es que su ciclo histerético sea altamente estable en presencia de deformaciones plásticas importantes (tanto máximas como acumuladas).

\section{Concepto}

La idea detrás de un contraviento desadherido consiste en fabricar un elemento estructural que trabaje a compresión sin que se pandee por efectos de esbeltez. Dado que los contravientos suelen trabajar de una manera estable a tensión, lo que se busca es un dispositivo capaz de disipar energía de manera estable en presencia de varias reversiones de carga. La Figura 1 muestra esquemáticamente el concepto de contraviento desadherido, e ilustra sus diferentes componentes: A) Una barra de acero que disipa energía a través de su extensión o contracción axial, B) Material confinante que restringe el pandeo del contraviento, y C) Camisa de acero que mantiene la integridad del material confinante y aporta mayor restricción contra pandeo. Bajo la acción de un sismo severo, se espera que sólo fluya el núcleo del contraviento (indicado en la figura).

El término desadherido se refiere al hecho de que la barra de acero se aísla del mortero o concreto confinante; es decir, que se impide contacto en la interface entre ambos materiales. Esto se hace con el fin de evitar que la resistencia a compresión de la barra sea significativamente mayor que su resistencia a tensión (Black et al. 2002, Uang y Nakashima 2003). La adherencia en los contravientos puede dar lugar a situaciones en las que el desequilibrio entre las fuerzas de compresión y tensión de dos contravientos que se intersecten en el centro del claro de una viga, induzca en ella fuerzas cortantes tan elevadas que dificulten el diseño de la viga, y que pueda resultar en un desempeño sísmico defíciente.

A pesar de que se logre una desadherencia perfecta, la barra será capaz de desarrollar mayor compresión que tensión, ya que la expansión del área de la sección transversal debida a la compresión y su contracción por tensión, son efectos que mecánicamente no pueden evitarse. Sin embargo, el desequilibro de las resistencias a compresión y tensión de la barra se reducen sustancialmente si se alivian los esfuerzos de fricción que se desarrollan en la interface entre barra y material confinante. Entre los materiales desadherentes que se han usado hasta el momento se tienen los siguientes (Uang y Nakashima 2003): pintura de asfalto, vinilos, cintas de espuma, caucho, silicón, teflón, y combinaciones de los materiales anteriores. El material desadherente debe: A) Evitar la fricción a través de aportar suficiente espacio para acomodar la expansión del área de la sección transversal de la barra de acero; B) Ser resistente al efecto de deslizamiento que se presente en el dispositivo por efectos de gravedad; C) Ser fácilmente adquirible; y D) Permitir su fácil colocación.

En cuanto al material confinante, suele usarse concreto o mortero. Dicho material debe: A) Tener resistencia para soportar los esfuerzos que la barra induzca en él; y B) Contar con aditivos expansivos y/o estabilizadores de volumen y un proceso de curado adecuado. Una discusión más detallada del concepto y uso de contravientos desadheridos puede encontrarse en Black et al. (2002), Uang y Nakashima (2003) y Tremblay et al. (2006). Las pruebas experimentales llevadas a cabo en contravientos desadheridos indican un comportamiento altamente estable ante la presencia de deformaciones plásticas severas, tanto unidireccionales como cíclicas. En Japón se han desarrollado varios tipos de contravientos desadheridos, y se cuenta con múltiples patentes (Watanabe et al. 1988, Uang y Nakashima 2003). De hecho, en ese país 
existen varias centenas de edificios cuyo sistema sismorresistente principal consiste en sistemas de contravientos desadheridos. En Taiwán, Canadá y EE.UU. también ha habido desarrollos experimentales de importancia y se han construido decenas de edificios que utilizan este tipo de dispositivo (Tremblay et al. 1999, Clark et al. 2000, Lopez et al. 2002, Ko et al. 2002, Mahin et al. 2004, Chen et al. 2004).

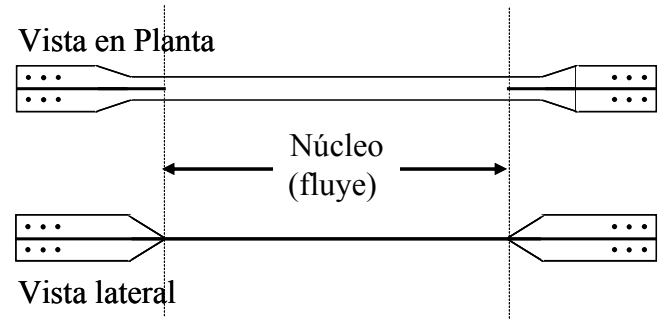

Tubo de acero

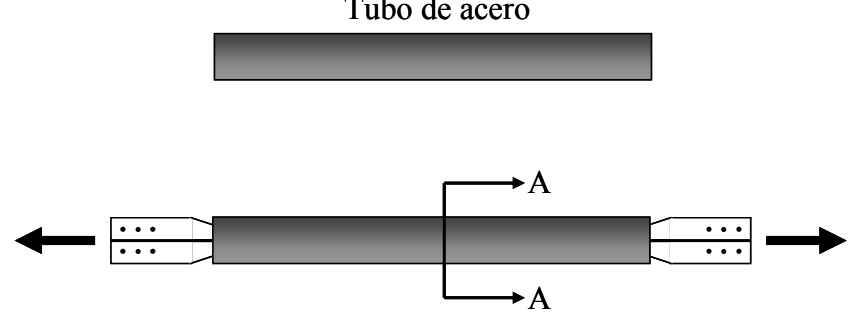

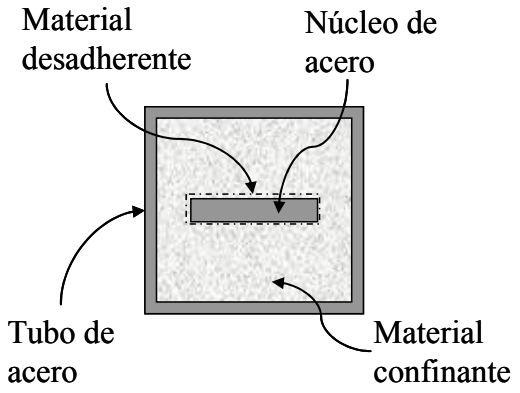

Corte A-A

Figura 1. Configuración esquemática de un contraviento desadherido (basado en Tremblay et al. 2006).

\section{Relaciones entre las propiedades estructurales y mecánicas de un contraviento}

En este artículo se plantea una metodología de diseño por desempeño para el dimensionado de un sistema de contravientos desadheridos. Se supondrá que la estructura sismorresistente por diseñarse consiste en una serie de marcos momentorresistentes encargados de soportar la carga gravitacional, a los cuales se añade un sistema de contravientos desadheridos encargado de tomar la totalidad de las cargas generadas por sismo. La metodología se basa en el planteamiento explícito del control del desplazamiento lateral de la edificación, lo que requiere de ayudas de diseño que permitan el diseño explícito de las propiedades estructurales de un contraviento en función de umbrales aceptables de desplazamiento y de las características mecánicas globales de diseño para el sistema de contravientos.

Es posible establecer una serie de relaciones explícitas entre las propiedades estructurales de un contraviento (ángulo de inclinación, área transversal y esfuerzo de fluencia) y las características mecánicas sismorresistentes que aporta a la edificación (rigidez lateral, resistencia lateral y desplazamiento lateral de fluencia). Cabe mencionar que en lo que sigue se desprecia la deformación global a flexión del sistema de contravientos, producto de la deformación axial de las columnas que los reciben; esto es, solo se considera la deformación global a corte producto de la deformación axial de los contravientos. Esto implica que las ayudas de diseño aquí desarrolladas no son aplicables a edificios esbeltos o de muchos pisos.

La rigidez lateral que aporta un contraviento a un entrepiso dado $\left(K_{L}\right)$ se relaciona con el área del núcleo $(A)$ por medio de la siguiente relación (Tremblay et al. 2006):

$\frac{K_{L}}{(A / L)}=\frac{E \cos ^{2} \theta}{\gamma+\eta(1-\gamma)}$ 
donde $L$ es la longitud total del contraviento, $E$ su módulo de elasticidad, y $\theta$ su ángulo de inclinación. $\gamma$ es el cociente entre la longitud del núcleo del contraviento ( $L_{c}$ conforme lo indica la Figura 2$)$ y $L$, y $\eta$ el cociente entre los esfuerzos axiales promedio fuera del núcleo y en el núcleo del contraviento. La Ecuación 1 hace posible establecer al área requerida de contravientos en un entrepiso en función de la geometría del sistema de contravientos y de la rigidez lateral que debe aportar al entrepiso.

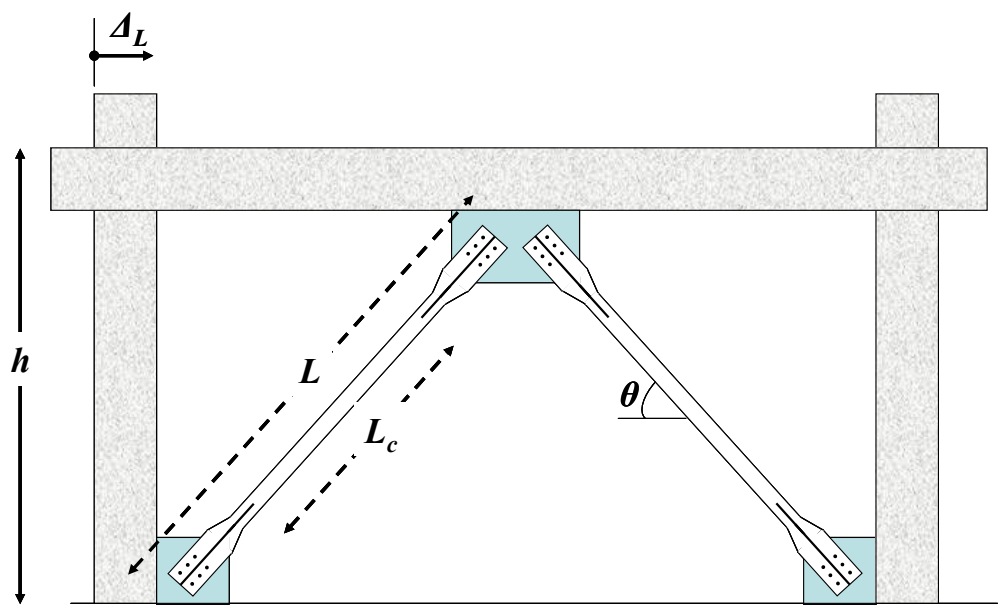

Figura 2. Notación utilizada para un contraviento y el entrepiso que lo recibe.

En cuanto a la distorsión de entrepiso en la que se presenta la fluencia de un contraviento, puede establecerse lo siguiente:

$$
\left(\frac{\Delta_{L}}{h}\right)_{y}=\frac{f_{y}[\gamma+\eta(1-\gamma)]}{E \operatorname{sen} \theta \cos \theta}
$$

donde $\Delta_{L}$ y $h$ son el desplazamiento lateral relativo y la altura del entrepiso, respectivamente (Figura 2); y $f_{y}$ el esfuerzo de fluencia del contraviento. El subíndice $y$ indica fluencia. La Ecuación 2 permite establecer el esfuerzo de fluencia requerido para los contravientos en función de la distorsión de entrepiso a la que debe fluir el sistema de contravientos. Para obtener mejores resultados de diseño, es importante que el esfuerzo de fluencia obtenido a partir de la Ecuación 2 corresponda al esfuerzo de fluencia esperado, y no a un valor reducido de diseño.

Finalmente, es posible establecer una relación entre el área del núcleo y el cortante lateral $\left(V_{L}\right)$ con que contribuye el contraviento al cortante lateral de entrepiso:

$V_{L}=A f_{y} \cos \theta$

Dentro de un contexto de diseño sísmico, la Ecuación 3 permite establecer el área requerida para el sistema de contravientos en función de la geometría del sistema de contravientos, del esfuerzo de fluencia esperado del acero y del cortante de entrepiso de diseño para dicho sistema. 


\section{ENFOQUE DE DISEÑO}

La aplicación de la metodología desarrollada en este artículo está basada en la concepción de una edificación cuyas cargas gravitacionales se resisten por medio de marcos de concreto reforzado, y cuya sismorresistencia esta aportada por un sistema de contravientos desadheridos que proporciona rigidez lateral, y que además funciona como sistema disipador de energía.

Bajo la acción de una excitación sísmica leve, se considera que el edificio exhibe un desempeño sísmico adecuado si satisface el estado límite de operación inmediata; lo que implica que tanto el sistema gravitacional como el sistema de contravientos no exhiban daño estructural de importancia. El sistema no estructural no debe exhibir daño alguno.

En cuanto al desempeño deseado para una excitación sísmica severa, se propone que el sistema destinado a soportar la carga gravitacional satisfaga el estado límite de operación inmediata, mientras que el sistema de contravientos desadheridos desarrolle comportamiento plástico de importancia que le permita disipar una elevada cantidad de energía. Este esquema se ilustra en la Figura 3, donde se indica que mientras el sistema gravitacional permanezca elástico, será capaz de aportar a la estructura contraventeada un endurecimiento por deformación significativo que estabiliza su respuesta dinámica y reduce de manera importante su deformación residual en presencia de demandas plásticas importantes (Uang y Kiggins 2003). Una vez que el sistema integrado se deforma más allá de su límite elástico, el daño se concentra en el sistema de contravientos. Después de la excitación sísmica, el daño se traduce en deformaciones residuales debido a la fluencia de los contravientos. Dado que el sistema gravitacional debe permanecer prácticamente elástico, las deformaciones residuales se eliminan una vez que se sustituyen los contravientos que fluyeron durante la excitación sísmica. Esto es, la rehabilitación estructural de la edificación consiste en sustituir los contravientos dañados. No debe haber colapso (parcial o total) del sistema no estructural.

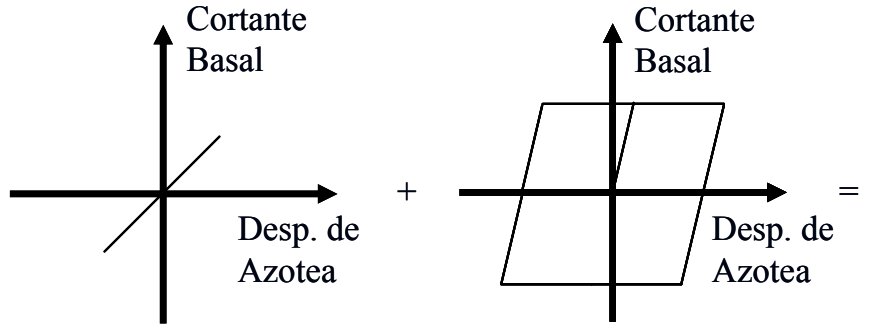

Sistema Gravitacional
Contravientos Desadheridos

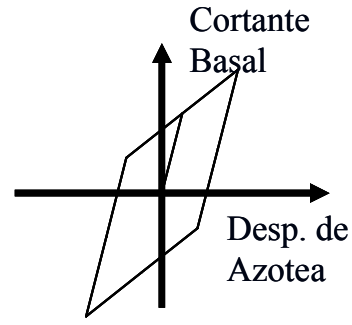

Sistemas integrados

Figura 3. Comportamiento propuesto para la edificación.

\section{METODOLOGÍA DE DISEÑO}

La Figura 4 resume esquemáticamente la metodología propuesta. Dicha metodología, que es aplicable al diseño de estructuras de ocupación estándar, considera los estados límite de servicio y seguridad de vida. El primer paso consiste en establecer una definición cualitativa del comportamiento deseado de la estructura. Esto se hace a través de la consideración explícita de los niveles de daño aceptable según los estados límite bajo consideración. El segundo paso consiste en la cuantificación del comportamiento deseado a través de establecer umbrales de respuesta global para la estructura con la ayuda de índices de daño. Durante el tercer paso, la metodología establece el valor de las características mecánicas de la estructura a nivel global a través de definir, con la ayuda de espectros de resistencia y 
desplazamiento, el valor de dos parámetros globales de diseño: el cortante basal, que cuantifica la resistencia lateral de diseño; y el periodo fundamental de vibración; que cuantifica los requerimientos de diseño de rigidez lateral. El dimensionado de los contravientos desadheridos se lleva a cabo con base en los valores establecidos para estos dos parámetros. Note que la metodología propuesta no maneja explícitamente la capacidad de deformación última del sistema de contravientos. Al respecto, se hace notar que mientras que las pruebas experimentales sugieren que un contraviento desadherido bien detallado es capaz de alcanzar deformaciones últimas muy elevadas, la metodología propuesta limita de manera importante la demanda de distorsión en el edificio para proteger al sistema gravitacional y a los elementos no estructurales. Bajo estas circunstancias, la capacidad de deformación de los contravientos no rige el diseño.

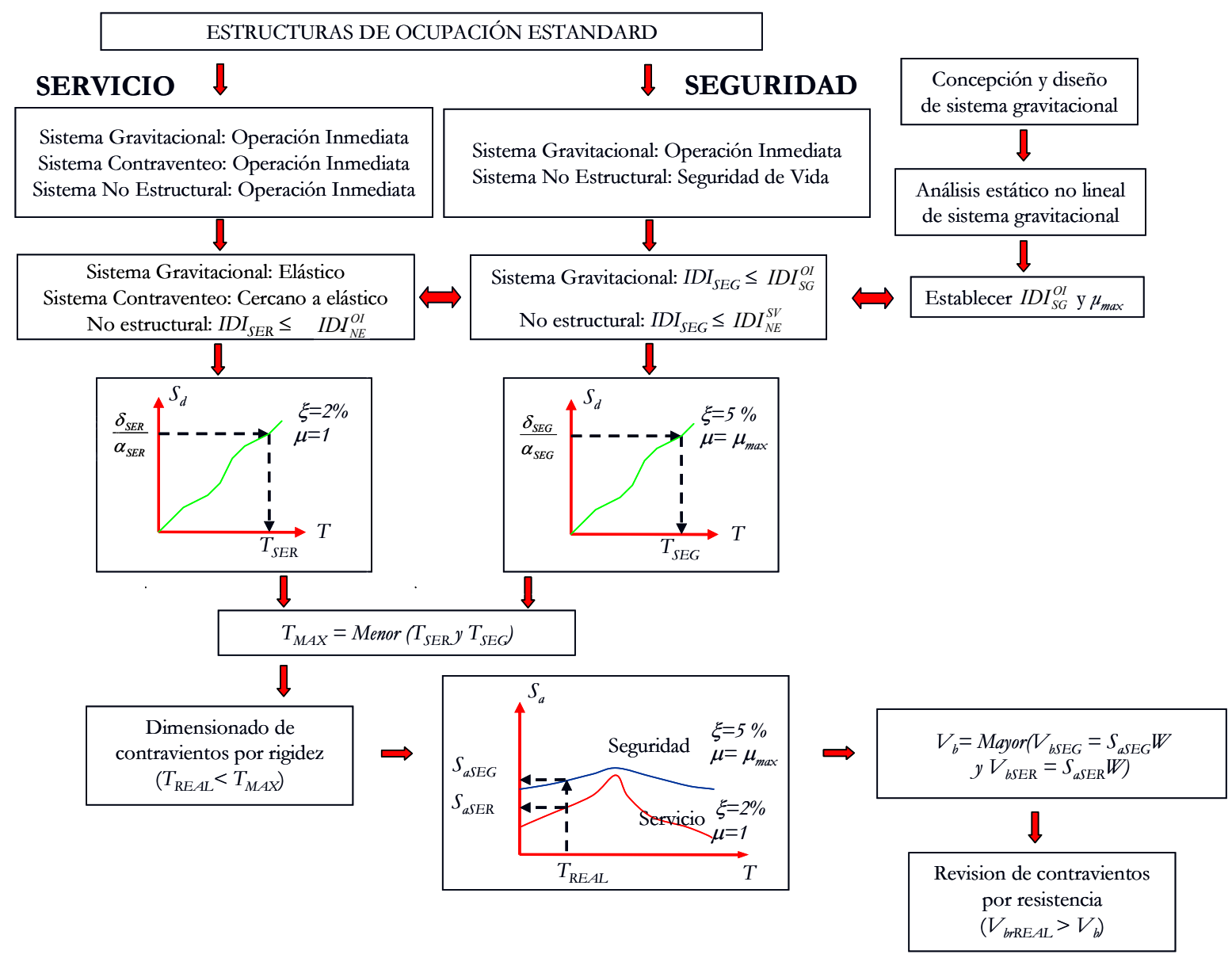

Figura 4. Metodología propuesta de diseño sísmico.

En cuanto a las definiciones cualitativas asociadas a ellos, los estados límite bajo consideración se consideran satisfechos si:

- Estado límite de servicio: La edificación debe satisfacer el criterio de operación inmediata. Tanto los contravientos desadheridos como el sistema que baja carga gravitacional pueden exhibir daño estructural leve (se acepta agrietamiento leve del sistema gravitacional y demandas leves de 
comportamiento plástico en los contravientos). Los elementos no estructurales deben permanecer sin daño alguno.

- Estado límite de seguridad: La edificación debe garantizar la integridad física de los ocupantes y ser fácilmente reparable. El sistema gravitacional debe ser capaz de operar al $100 \%$ de su capacidad después del sismo (operación inmediata), lo que implica que sus elementos estructurales exhiban leves demandas de comportamiento plástico. El sistema de contravientos desadheridos debe desarrollar comportamiento plástico de importancia que le permita disipar un porcentaje elevado de la energía que la excitación sísmica induce en la estructura. En cuanto a los elementos no estructurales, debe evitarse su colapso local.

En cuanto a la cuantificación del estado límite de servicio, se considera que el sistema gravitacional y los contravientos satisfacen sus criterios de desempeño estructural mientras permanezcan elásticos y desarrollen leves demandas de comportamiento plástico, respectivamente; y que el daño en los elementos no estructurales se controla de manera adecuada si a su vez se controla la distorsión máxima de entrepiso para el estado límite de servicio $\left(I D I_{S E R}\right)$ dentro del umbral de distorsión que garantice la operación inmediata de los elementos no estructurales $\left(I D I_{N E}^{O I}\right.$ ). Reyes (1999) ofrece, en función del tipo de elemento no estructural y de la manera en que este se conecta a la estructura, umbrales de distorsión asociados al inicio de daño y al daño total.

El estado límite de seguridad de vida se satisface si se controla la distorsión máxima de entrepiso $\left(I D I_{S E G}\right)$ de acuerdo con lo siguiente: 1) Que el daño estructural en el sistema gravitacional debe controlarse a través de establecer un umbral a la distorsión de entrepiso que garantice su operación inmediata ( $\left.I D I_{S G}^{O I}\right)$, y 2) Que el daño no estructural debe controlarse a través de establecer un umbral para la distorsión máxima que prevenga colapso local en elementos no estructurales ( $\left.I D I_{N E}^{S V}\right)$.

El diseño numérico inicia a través de la concepción y diseño del sistema gravitacional. El sistema gravitacional se diseña para resistir exclusivamente las cargas gravitacionales, sin consideración explícita de las cargas laterales. Se recomienda utilizar para los marcos del sistema gravitacional detallado estándar, lo que corresponde al detallado asociado a un factor de comportamiento sísmico $(Q)$ de 2 . Una vez establecido el sistema gravitacional, se lleva a cabo un análisis estático no lineal bajo deformación lateral monótonamente creciente del mismo, y se establece la distorsión de entrepiso asociada a su estado límite de operación inmediata $\left(I D I_{S G}^{O I}\right.$ ). Conforme a las indicaciones del FEMA 356 (Federal Emergency Management Agency 1997), se define la distorsión de entrepiso para operación inmediata como aquella en que la máxima demanda de rotación plástica en los elementos estructurales de los marcos gravitacionales sea de 0.005. Además de lo anterior, el proceso de diseño numérico requiere estimar de manera aproximada la máxima demanda de ductilidad asociada al sistema de contravientos desadheridos $\left(\mu_{\max }\right)$. Este valor es particularmente importante porque como se comentará más adelante, se utiliza para definir los espectros de desplazamiento y resistencia utilizados para el estado límite de seguridad. Una aproximación razonable del valor de $\mu_{\max }$ para una estructura regular de pocos pisos puede obtenerse a partir del cociente $I D I_{S E G} / I D I_{y}$; donde $I D I_{y}$ representa la distorsión de entrepiso a la cual debe fluir el sistema de contravientos (ver Ecuación 2), e $I D I_{S E G}$ la demanda máxima de distorsión permisible en la edificación.

El valor del primer parámetro de diseño, que es el periodo fundamental de vibración del edificio, se establece conforme a lo ilustrado en la Figura 4. El umbral de distorsión para un estado límite puede ser usado para establecer el umbral de desplazamiento lateral de azotea que la edificación puede alcanzar para dicho estado límite: 


$$
\begin{gathered}
\delta_{S E R}=\frac{I D I_{S E R} H}{C O D_{S E R}} \\
\delta_{S E G}=\frac{I D I_{S E G} H}{C O D_{S E G}}
\end{gathered}
$$

donde $H$ es la altura total del edificio a partir del nivel de suelo, y $C O D$ un coeficiente de distorsión que contempla que la distorsión no es constante en todos los entrepisos del edificio. En particular, $C O D$ cuantifica la relación existente entre la distorsión máxima de entrepiso y la distorsión promedio en la estructura (Qi y Moehle 1991). Con base en lo discutido por Qi y Moehle (1991) y Bertero et al. (1991), y en los estudios llevados a cabo por Teran (2004), la Tabla 1 presenta valores sugeridos de COD para el prediseño de estructuras regulares que exhiben un comportamiento global del tipo viga de corte.

Tabla 1. Rangos sugeridos para el valor de $C O D$

\begin{tabular}{ccc}
\hline Ductilidad & \multicolumn{2}{c}{$C O D$} \\
\cline { 2 - 3 } Global & Mínimo & Máximo \\
\hline 1 & 1.2 & 1.5 \\
$2+$ & 1.5 & 2.0 \\
\hline
\end{tabular}

A partir de los umbrales de desplazamiento de azotea para los estados límites de servicio y seguridad ( $\delta_{S E R}$ y $\delta_{S E G}$, respectivamente) y espectros de desplazamiento para dichos estados límite, puede estimarse el periodo máximo de la estructura. Note que antes de utilizar los valores de $\delta_{S E R}$ y $\delta_{S E G}$, es necesario considerar que la edificación es un sistema de varios grados de libertad, y que un espectro de desplazamientos resume resultados derivados de sistemas de un grado de libertad. Conforme a lo mostrado en la Figura 4, los valores de $\delta$ deben modificarse para contemplar el efecto de varios grados de libertad a través del parámetro $\alpha$. Con base en las recomendaciones del FEMA 306 (Applied Technology Council 1998) y los resultados presentados por Terán (2004), la Tabla 2 presenta valores sugeridos de $\alpha$ para el prediseño de estructuras regulares que exhiben un comportamiento global del tipo viga de corte.

Tabla 2. Valores sugeridos para $\alpha$

\begin{tabular}{ccc}
\hline Número de pisos & \multicolumn{2}{c}{$\alpha$} \\
\cline { 2 - 3 } & $\mu=1$ & $\mu=2+$ \\
\hline 1 & 1.0 & 1.0 \\
2 & 1.2 & 1.1 \\
3 & 1.3 & 1.2 \\
$5+$ & 1.4 & 1.2 \\
\hline
\end{tabular}

En congruencia con lo planteado para el daño estructural aceptable para el estado límite de servicio, el espectro de desplazamientos de servicio contempla comportamiento elástico y un porcentaje de amortiguamiento crítico $(\xi)$ de $2 \%$. Para el caso del estado límite de seguridad, se plantea el uso de un espectro de desplazamientos para ductilidad máxima de $\mu_{\max }$ y $\xi$ de $5 \%$. En cuanto a los valores de porcentaje de amortiguamiento crítico asignados a los espectros, es importante que dicho porcentaje sea congruente con el nivel de esfuerzos esperado en los elementos estructurales. Chopra (2001) sugiere que el amortiguamiento tiende a crecer de manera importante conforme el nivel de esfuerzos en un elemento estructural se incrementa. En particular, si el nivel de esfuerzos es del orden o menor que el $50 \%$ del esfuerzo de fluencia, el coeficiente equivalente de amortiguamiento puede ser sustancialmente menor al 5\%; mientras que si el nivel de esfuerzos corresponde a la fluencia del elemento estructural, el amortiguamiento suele ser mayor que $5 \%$. Se considera que los porcentajes de $2 \%$ y $5 \%$ asociados a los 
estados límite de servicio y seguridad, respectivamente, son valores razonablemente conservadores asociados al estado de daño estructural contemplado por cada uno de dichos estados límite.

La Figura 4 indica que el valor de periodo fundamental de vibración $\left(T_{M A X}\right)$ para el que debe diseñarse la edificación corresponde al menor de los valores dados por $T_{S E R}$ y $T_{S E G}$, que a su vez corresponden a los valores de periodo que satisfacen los requerimientos de diseño impuestos por los estados límite de servicio y seguridad, respectivamente. Se destaca que se utiliza el menor periodo como valor de diseño porque este resulta en los mayores requerimientos de rigidez lateral. Una vez que se establece el valor de $T_{M A X}$, se procede a dimensionar (definir el área de) los contravientos. Una vez que los contravientos tengan un área tal que el periodo fundamental de vibración estimado para la edificación $\left(T_{R E A L}\right)$ sea igual o ligeramente menor que $T_{M A X}$, se determina el valor del segundo parámetro de diseño: el cortante basal. Como se muestra, el cortante basal de diseño corresponde al mayor de los cortantes establecidos para los estados límite de servicio y seguridad ( $V_{b S E R}$ y $V_{b S E G}$, respectivamente). Note que en el caso del estado límite de servicio, el cortante basal se estima a partir de evaluar para $T_{R E A L}$ un espectro elástico de pseudo-aceleración para $\xi$ de 0.02 ; y que el correspondiente a seguridad de vida se obtiene al evaluar, para ese mismo valor de periodo, un espectro correspondiente a $\mu_{\max } \mathrm{y} \xi$ de 0.05 . En caso de una estructura de pocos niveles es posible utilizar el valor del cortante basal de diseño y el método estático de análisis para establecer las fuerzas laterales de diseño que se utilizan para revisar la capacidad resistente de los contravientos. De requerirse podrían llevarse a cabo análisis dinámicos modales de la edificación con ambos espectros de resistencia, y revisar la resistencia de los contravientos a partir de la condición crítica que surja a partir de ambos análisis.

En caso de que el área de contravientos establecida por cuestiones de rigidez sea incapaz de acomodar las demandas de resistencia requeridas por la estructura, se ajustan. Es importante hacer notar que después de que se diseñan los contravientos, debe adecuarse el sistema gravitacional para recibirlos, y que deben utilizarse conceptos de diseño por capacidad con este fin.

\section{SISTEMA GRAVITACIONAL}

Para la edificación bajo consideración, dicho sistema consiste en marcos de concreto reforzado diseñados explícitamente para tomar las cargas gravitacionales de acuerdo a la versión 2004 del Reglamento de Construcciones del Distrito Federal (RCDF) y sus Normas Técnicas Complementarias para Diseño y Construcción de Estructuras de Concreto (NTCDCEC).

\section{Estructuración}

La Figura 5 muestra la geometría de los marcos del edificio, el cual se considera ubicado en la Zona del Lago del D. F. El diseño estructural de los marcos considera exclusivamente las cargas gravitacionales actuantes en la estructura (viva y muerta) y un detallado estándar ( $Q$ de 2$)$. Lo anterior resulta en una estructura muy ligera en peso, con un contenido bajo de acero, y un detallado relativamente simple.

En cuanto a los materiales estructurales, se consideró una resistencia a compresión del concreto $\left(f_{c}^{\prime}\right)$ de $250 \mathrm{~kg} / \mathrm{cm}^{2}$, y un esfuerzo de fluencia para el acero $\left(f_{y}\right)$ de $4200 \mathrm{~kg} / \mathrm{cm}^{2}$. De acuerdo al análisis de cargas gravitacionales, las cargas máximas por unidad de área para azotea y entrepiso resultan iguales a $0.580 \mathrm{ton} / \mathrm{m}^{2}$ y $0.762 \mathrm{ton} / \mathrm{m}^{2}$, respectivamente. Las masas de azotea y entrepiso, estimadas a partir de la carga viva instantánea, resultaron iguales a $130.22 \mathrm{~kg}-\mathrm{seg}^{2} / \mathrm{cm}$ y $187.27 \mathrm{~kg}-\mathrm{seg}^{2} / \mathrm{cm}$, respectivamente.

El peralte de la losa de $15 \mathrm{~cm}$ se estableció conforme a los requerimientos de la Sección 6.3.3.3 de las NTCDCEC. El refuerzo de la losa consta de barras del \#3 @ $25 \mathrm{~cm}$ en ambas direcciones. El control 
de deflexiones y de agrietamiento rigió el dimensionado de la losa y las vigas. Durante los análisis se utilizó el momento de inercia de la sección agrietada de los elementos estructurales. Al respecto, se utilizó un momento de inercia para las vigas igual al 50\% del momento de inercia de la sección gruesa; el porcentaje para el caso de columnas fue de $70 \%$. Se diseñaron dos marcos del edificio, uno externo y uno interno.

Todas las vigas del edificio exhiben la misma cuantía de acero y el mismo detallado. La Figura 6 muestra un corte transversal con las dimensiones y detallado de los extremos de una de las vigas centrales del edificio. Las columnas se diseñaron por flexocompresión, de tal manera que pudieran acomodar el momento flexionante y carga axial inducidas en ellas por las cargas gravitacionales. Se consideró conveniente por razones constructivas, que todas las columnas en el edificio tuvieran la misma sección transversal. En cuanto a la cuantía de acero longitudinal, rigió la mínima, que corresponde al $1 \%$ del área transversal. La Figura 7 muestra la sección transversal de las columnas.

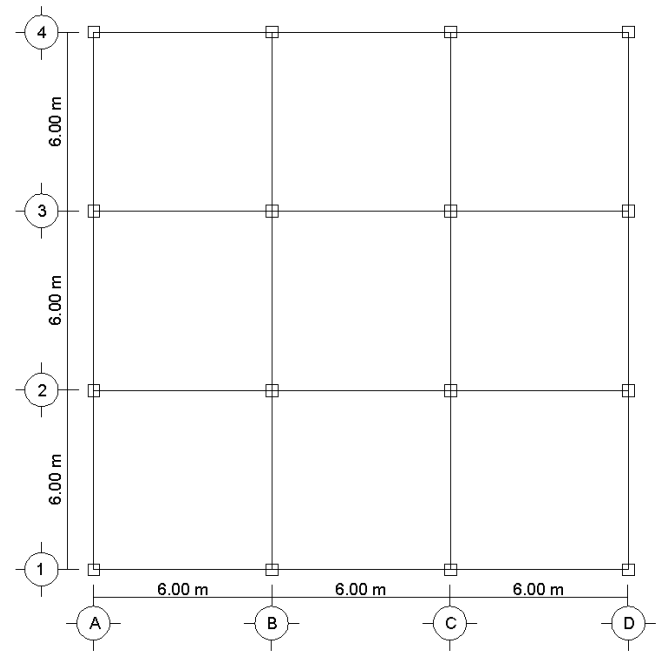

a) Planta

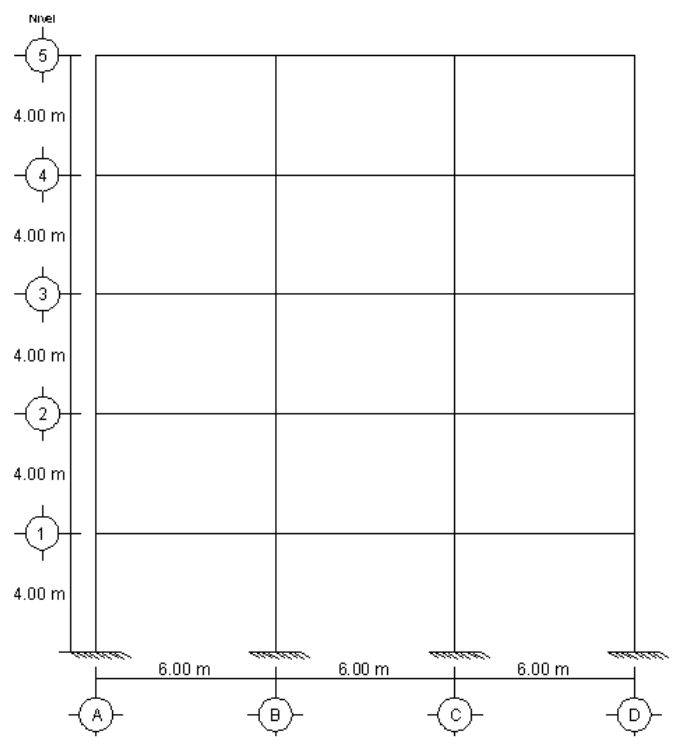

b) Elevación

Figura 5. Configuración estructural de los marcos del edificio.

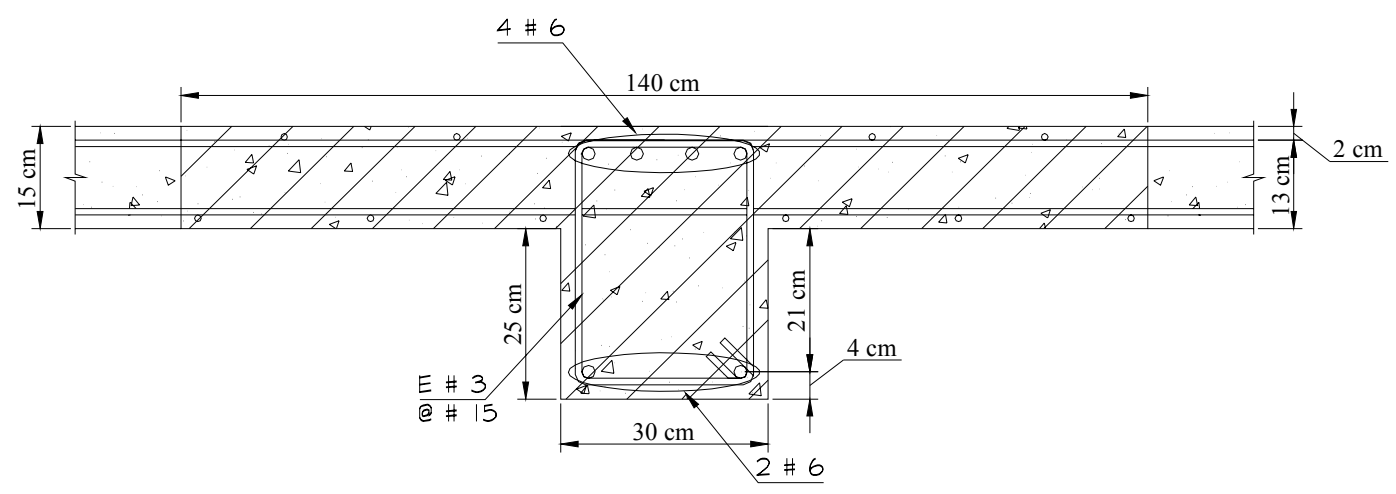

Figura 6. Dimensiones y refuerzo en los extremos de las vigas. 


\section{Características mecánicas y dinámicas}

Una vez concluido el diseño del sistema gravitacional, se procedió a estimar sus características mecánicas y dinámicas a partir de un modelo de análisis no lineal. En resumen, se estableció un modelo plano (bidimensional) que consideró un marco interno y un marco externo. Se contempló explícitamente el nivel esperado de agrietamiento en las vigas y columnas, así como el efecto que la losa tiene en la resistencia, rigidez y capacidad de deformación de las vigas. Se utilizaron modelos analíticos bien conocidos para establecer las curvas esfuerzo-deformación para el concreto confinado y no confinado, así como para el acero. A partir de estas curvas y de suponer que una sección plana permanece plana después de la flexión, se establecieron con el programa RESPONSE 2000 (Bentz y Collins 2000) las curvas momento-curvatura en ambos extremos de los elementos estructurales. Luego se establecieron idealizaciones bilineales de estas curvas por medio de definir curvaturas de fluencia y última. La resistencia, rigidez y capacidad de deformación de los elementos estructurales se establecieron directamente de estas curvas idealizadas.

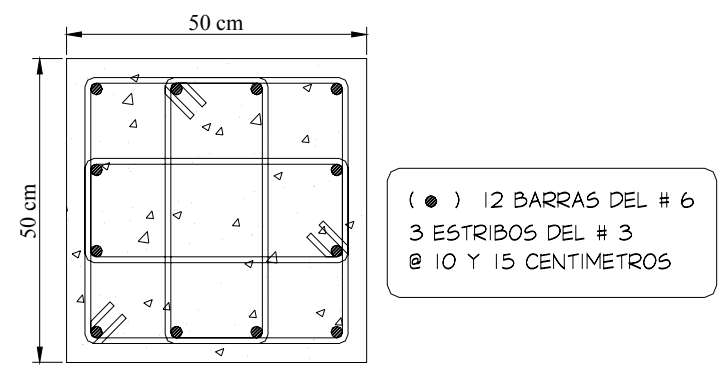

Figura 7. Dimensiones y refuerzo de las columnas.

La porción de la losa que interactúa, tanto en tensión como compresión, con las vigas se definió a partir de las recomendaciones de Pantazopoulou y French (2001) para una distorsión de 2\%. Cabe mencionar que las propiedades de los materiales estructurales utilizadas para estimar las propiedades estructurales de vigas y columnas no son las de diseño, sino las esperadas. En cuanto a esto, se utilizaron las recomendaciones del FEMA 356 para establecer las propiedades estructurales del concreto y del acero. Las columnas del primer piso se modelaron como empotradas en la base, y se consideró tanto el endurecimiento por deformación en las vigas como los efectos de segundo orden derivados de la carga gravitacional. Finalmente, el modelo contempló las zonas de rigidez infinita en los extremos de los elementos estructurales.

Una herramienta útil para evaluar las características mecánicas globales de una estructura es un análisis estático bajo deformación lateral monótonamente creciente (pushover). Este tipo de análisis consiste en aplicar una serie de cargas laterales con valor relativo constante en altura, hasta alcanzar una deformación objetivo. En este estudio se utilizó un patrón de cargas triangulares. El análisis estático no lineal se llevó a cabo con el programa DRAIN 2DX (Prakash et al. 1993). Entre los resultados relevantes arrojados por este tipo de análisis está la curva cortante basal contra desplazamiento de azotea, la evolución de las distorsiones de entrepiso y del daño local en función del desplazamiento de azotea, y la descripción del mecanismo plástico que desarrolla la estructura.

Acorde al análisis estático no lineal, el daño tiende a concentrarse en las vigas de los marcos, aunque se forma un número importante de articulaciones plásticas en las columnas, particularmente en las ubicadas en la planta baja. 
La Figura 8 muestra para el sistema gravitacional la curva cortante basal $\left(V_{b}\right)$ contra desplazamiento de azotea $(\delta)$. Aunque el modelo de análisis contempla dos de los cuatro marcos del edificio, los resultados que se presentan corresponden a la totalidad de la estructura. El trabajo conjunto de los marcos resulta en un cortante basal de 80 toneladas, lo que corresponde a $9 \%$ del peso de la edificación. Puede observarse el efecto perjudicial de los efectos $P-A$, particularmente para los marcos internos. Los marcos exhiben un comportamiento prácticamente elástico hasta un desplazamiento de azotea de $7 \mathrm{~cm}, \mathrm{y}$ responden de manera estable hasta un desplazamiento de azotea de $10 \mathrm{~cm}$. El valor del periodo fundamental de vibración estimado para el edificio resultó de $1.44 \mathrm{seg}$.

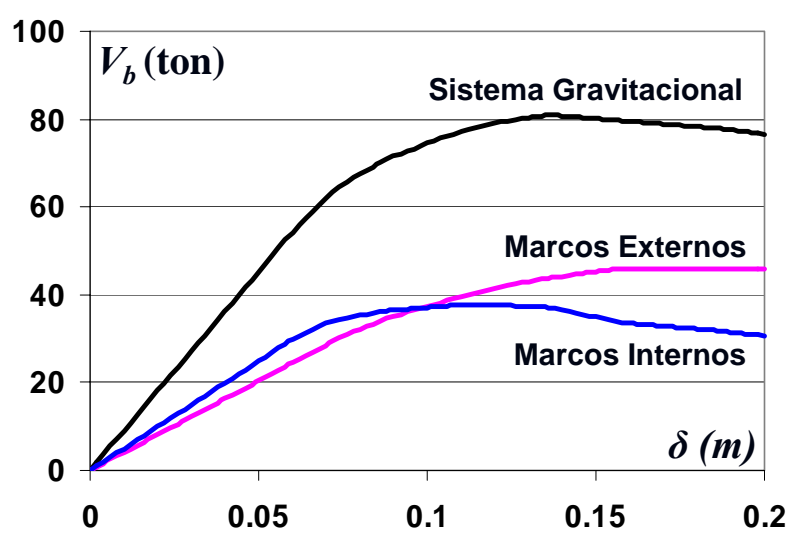

Figura 8. Curva cortante basal contra desplazamiento de azotea, sistema gravitacional.

La Figura 9 muestra la evolución de la distorsión de entrepiso para los diferentes niveles del edificio conforme se incrementa el desplazamiento lateral de azotea. Los desplazamientos de azotea $(\delta)$ indicados en la figura están en centímetros. La deformación lateral de la estructura tiende a concentrarse en los pisos intermedios, particularmente en los niveles 2 y 3. Note en la Figura 8 que el edificio empieza a exhibir comportamiento no lineal a partir de un desplazamiento de azotea de entre 8 y $9 \mathrm{~cm}$, lo que de acuerdo con la Figura 9 corresponde a una distorsión cercana a 0.006. Este valor de distorsión es consistente con el valor de 0.0073, identificado por Reyes (1999) como el umbral a partir del cual empiezan a dañarse los elementos estructurales de un marco no dúctil de concreto reforzado.

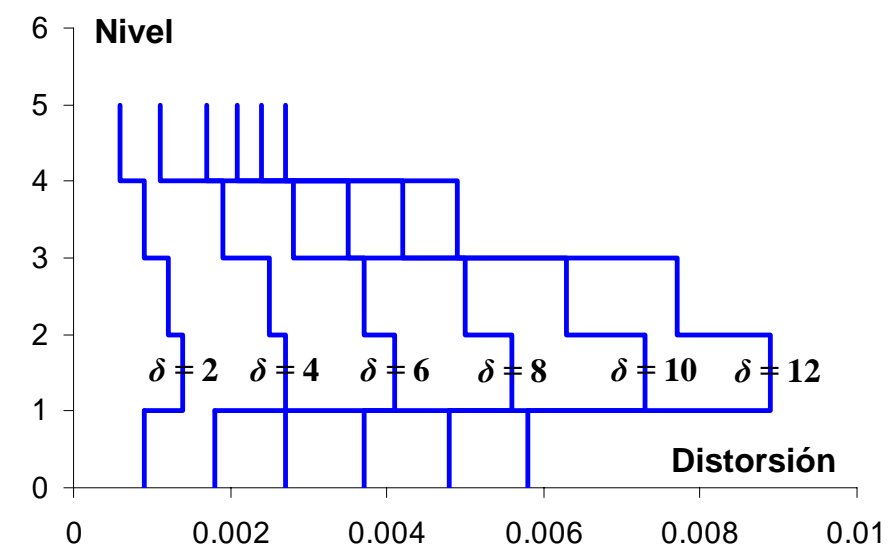

Figura 9. Distorsiones de entrepiso en función del desplazamiento de azotea, sistema gravitacional. 
El documento FEMA 356 establece que los elementos estructurales de un marco de concreto reforzado sin un buen detallado sísmico pueden acomodar rotaciones plásticas del orden de 0.005 para el estado límite de operación inmediata. Con este estado límite en mente, se evaluaron los valores de desplazamiento de azotea y distorsión de entrepiso correspondientes a la formación de una rotación plástica de 0.005 para el elemento estructural crítico. De acuerdo a los resultados obtenidos del análisis, la primera viga en alcanzar dicha rotación lo hace para un desplazamiento de azotea de $11.4 \mathrm{~cm}$ y una distorsión de entrepiso cercana a 0.0084. A partir de esto, se define el umbral de distorsión para el sistema gravitacional:

$I D I_{S G}^{O I} \leq 0.0084$

Note que el sistema gravitacional de la edificación bajo consideración consiste en marcos muy ligeros de concreto reforzado, con una cuantía baja de acero longitudinal y un detallado simple. Como consecuencia, el sistema gravitacional exhibe resistencia y rigidez laterales muy por debajo de lo requerido por un sistema sismorresistente.

\section{DISEÑO DEL SISTEMA DE CONTRAVIENTOS}

\section{Cuantificación del desempeño requerido}

En cuanto a los elementos no estructurales del edificio, se considera que estos están constituidos por muros de tablarroca unidos a la estructura a través de clavarlos y pegarlos a un marco de madera, fijado a su vez a la estructura. Reyes (1999) indica que mientras que la distorsión de entrepiso que inicia daño en este tipo de elementos es 0.003 , la distorsión para daño total corresponde a un valor de 0.008 .

Considerando que el Apéndice A de las Normas Técnicas Complementarias para Diseño por Sismo del Reglamento de Construcciones para el D.F. indican que, por razones no estructurales, la distorsión de entrepiso debe limitarse para el estado límite de servicio a 0.002, se proponen los siguientes umbrales de distorsión para los elementos no estructurales:

$I D I_{N E}^{O I} \leq 0.002$

$I D I_{N E}^{S V} \leq 0.008$

donde $I D I_{N E}^{O I}$ y $I D I_{N E}^{S V}$ son los valores máximos permisibles para la distorsión de entrepiso correspondiente a los estados límite de operación inmediata y de seguridad de vida, respectivamente, para los elementos no estructurales. Si conforme a lo requerido por la Figura 4 se superponen las consideraciones para el desempeño estructural del sistema gravitacional ( $I D I_{S G}^{O I}$, Ecuación 5) y para el desempeño no estructural del edificio ( $I D I_{N E}^{O I}$ y $I D I_{N E}^{S V}$, Ecuaciones 6 y 7), las distorsiones críticas de diseño son:

$I D I_{\text {SER }} \leq 0.002$

$I D I_{S E G} \leq$ menor $(0.008$ y 0.0084$)=0.008$ 
Para que satisfaga sus condiciones de desempeño, es necesario concebir al sistema de contravientos para que fluya a distorsiones cercanas a 0.002 , y para exhibir comportamiento plástico de consideración para distorsiones cercanas a 0.008. Acorde a la Ecuación 2, y considerando que: A) La geometría de los contravientos y sus conexiones es tal que $\gamma=0.5$ y $\eta=0.333$; B) El sistema de contravientos debe fluir a distorsiones cercanas a 0.002 ; y C) La configuración estructural y la ubicación del sistema de contravientos es tal que $\theta=53.13^{\circ}$; se tiene:

$$
f_{y}=\left(\frac{\Delta_{L}}{h}\right)_{y} \frac{2^{\prime} 000,000 \operatorname{sen} 53.13^{\circ} \cos 53.13^{\circ}}{0.5+0.333(1-0.5)}=2880 \mathrm{~kg} / \mathrm{cm}^{2}
$$

Para la edificación bajo consideración se decide que es aceptable que el sistema de contravientos exhiba ligeras demandas de comportamiento plástico para el estado límite de servicio, de tal manera que se utiliza un acero con un $f_{y}$ esperado de $2375 \mathrm{~kg} / \mathrm{cm}^{2}$. Esto resulta en que la distorsión real de fluencia para dicho sistema sea (Ecuación 2):

$$
\left(\frac{\Delta_{L}}{h}\right)_{y}=\frac{2375[0.5+0.333(1-0.5)]}{2^{\prime} 000,000 \operatorname{sen} 53.13^{o} \cos 53.13^{\circ}}=0.00165
$$

Considerando que la máxima distorsión de entrepiso permisible durante el estado límite de seguridad es de 0.008 (Ecuación 9), el sistema de contravientos debe ser capaz de desarrollar una ductilidad máxima de entrepiso $\left(\mu_{e}\right)$ cercana a $\frac{0.008}{0.00165}=4.8$. Dado que el valor de ductilidad máxima que debe asignarse a la edificación debe ser menor que la ductilidad máxima de entrepiso (Chopra 2001), y que la edificación bajo consideración tiene pocos pisos, se establece una ductilidad máxima $\left(\mu_{\max }\right)$ de 4 para el estado límite de seguridad. Es posible demostrar que la ductilidad demandada en cada contraviento es igual a la ductilidad de entrepiso, y que la demanda de ductilidad en el núcleo del contraviento desadherido $\left(\mu_{c}\right)$ está dada por:

$\mu_{c}=\left[1+\frac{\eta}{\gamma}(1-\gamma)\right]\left(\mu_{e}-1\right)+1$

La Tabla 3 Resume valores de $\mu_{c}$ para diferentes combinaciones de valores de $\eta, \gamma$ y $\mu_{e}$. Note que para los valores de $\eta, \gamma$ y $\mu_{e}$ utilizados aquí $(0.333,0.5$ y 4.8 , respectivamente), se obtiene una ductilidad local cercana a 6, valor muy inferior al que puede ser acomodado por el acero del núcleo. Tan solo como referencia, las pruebas experimentales indican que un contraviento desadherido puede desarrollar de manera estable ductilidades de entrepiso mayores que diez. Como se mencionó anteriormente, la metodología propuesta limita de manera importante la demanda de distorsión en el edificio para proteger al sistema gravitacional y a los elementos no estructurales; de tal manera que no es necesario revisar la ductilidad local de los contravientos ya que su capacidad de deformación no rige el diseño.

\section{Excitaciones sísmicas de diseño}

Para la elaboración de espectros de diseño se consideraron dos grupos de acelerogramas registrados durante diferentes eventos sísmicos en la Zona del Lago del Distrito Federal. El primero grupo de acelerogramas, correspondiente al estado límite de servicio, contiene los movimientos resumidos en la Tabla 4, los cuales fueron escalados de tal forma que su velocidad máxima fuera igual a la sexta parte de la velocidad máxima correspondiente al acelerograma registrado durante 1985 en la dirección este-oeste 
de la Secretaría de Comunicaciones y Transporte (SCTEO). El segundo grupo, correspondiente al estado límite de seguridad, se resume en la Tabla 5. Los movimientos contenidos en el segundo grupo se escalaron de tal manera que la velocidad máxima de cada registro fuera igual a la velocidad máxima correspondiente a SCTEO. Los espectros de diseño de un grupo dado se establecieron a partir de la media más una desviación estándar de los correspondientes espectros derivados de cada acelerograma dentro de dicho grupo. En las tablas, $T_{g}$ denota el periodo dominante del acelerograma.

Tabla 3. Demandas de ductilidad en el núcleo de un contraviento desadherido en función de sus propiedades geométricas y la ductilidad de entrepiso

\begin{tabular}{cccccccc}
\hline \multirow{2}{*}{$\mu_{e}$} & \multicolumn{3}{c}{$\eta=0.333$} & \multicolumn{3}{c}{$\eta=0.5$} \\
\cline { 2 - 4 } \cline { 6 - 8 } & $\gamma=1.0$ & $\gamma=0.5$ & $\gamma=0.25$ & & $\gamma=1.0$ & $\gamma=0.5$ & $\gamma=0.25$ \\
\hline 2 & 2 & 2.33 & 3 & & 2 & 2.5 & 3.5 \\
3 & 3 & 3.67 & 5 & & 3 & 4 & 6 \\
4 & 4 & 5 & 7 & & 4 & 5.5 & 8.5 \\
5 & 5 & 6.33 & 9 & & 5 & 7 & 11 \\
6 & 6 & 7.67 & 11 & & 6 & 8.5 & 13.5 \\
\hline
\end{tabular}

Tabla 4. Acelerogramas correspondientes al estado límite de servicio

\begin{tabular}{ccccc}
\hline \multirow{2}{*}{ Identificación } & Lugar de registro & Fecha & Factor de escala & $T_{g}(\mathrm{seg})$ \\
\hline$s 31$ & CUPJ EO & $14 / 09 / 1995$ & 1.317 & 2 \\
$s 32$ & CUPJ NS & $14 / 09 / 1995$ & 1.542 & 2 \\
$s 43$ & Garibaldi EO & $10 / 12 / 1994$ & 1.891 & 2 \\
$s 45$ & Garibaldi EO & $14 / 09 / 1995$ & 1.047 & 2 \\
$s 46$ & Garibaldi NS & $14 / 09 / 1995$ & 1.266 & 2 \\
$s 51$ & Hospital Juárez EO & $14 / 09 / 1995$ & 0.852 & 2 \\
$s 59$ & Liverpool EO & $09 / 10 / 1995$ & 1.644 & 2 \\
$s 77$ & Tlatelolco EO & $10 / 12 / 1994$ & 2.222 & 2.1 \\
$s 79$ & Tlatelolco EO & $14 / 09 / 1995$ & 1.289 & 2 \\
$s 89$ & Alameda EO & $14 / 09 / 1995$ & 0.966 & 2 \\
$s 90$ & Alameda NS & $14 / 09 / 1995$ & 1.322 & 2 \\
$s 119$ & C. U Juárez EO & $10 / 12 / 1994$ & 2.278 & 1.9 \\
$s 125$ & Cibeles EO & $24 / 10 / 1993$ & 2.652 & 2.1 \\
$s 129$ & Cibeles EO & $09 / 10 / 1995$ & 1.993 & 2 \\
$s 131$ & Angares EO & $14 / 09 / 1995$ & 1.072 & 1.6 \\
$s 143$ & Tlatelolco EO & $14 / 09 / 1995$ & 1.216 & 2 \\
$s 144$ & Tlatelolco NS & $14 / 09 / 1995$ & 1.726 & 1.9 \\
\hline
\end{tabular}

Tabla 5. Acelerogramas correspondientes al estado límite de seguridad

\begin{tabular}{ccccc}
\hline \multirow{2}{*}{ Identificación } & Lugar de registro & Fecha & $\begin{array}{c}\text { Factor de } \\
\text { escala }\end{array}$ & $T_{g}(\mathrm{seg})$ \\
\hline$m x 01$ & Alameda EO & $04 / 25 / 89$ & 4.046 & 2.1 \\
$m x 03$ & Garibaldi EO & $04 / 25 / 89$ & 3.491 & 2.2 \\
$m x 04$ & Tlahuac EO & $09 / 19 / 85$ & 1.766 & 2.1 \\
$m x 06$ & Tlahuac NS & $09 / 21 / 85$ & 4.664 & 2.0 \\
$m x 07$ & Tlahuac EO & $09 / 21 / 85$ & 4.046 & 1.9 \\
$m x 08$ & SCT EO & $09 / 19 / 85$ & 1 & 2.0 \\
\hline
\end{tabular}




\section{Dimensionado de contravientos}

La Figura 10 muestra la configuración estructural y ubicación de los contravientos en la estructura. En resumen, se contraventea la crujía central de los dos marcos externos. Conforme a las dimensiones de los entrepisos, el ángulo de inclinación de los contravientos es de $53.13^{\circ}$.

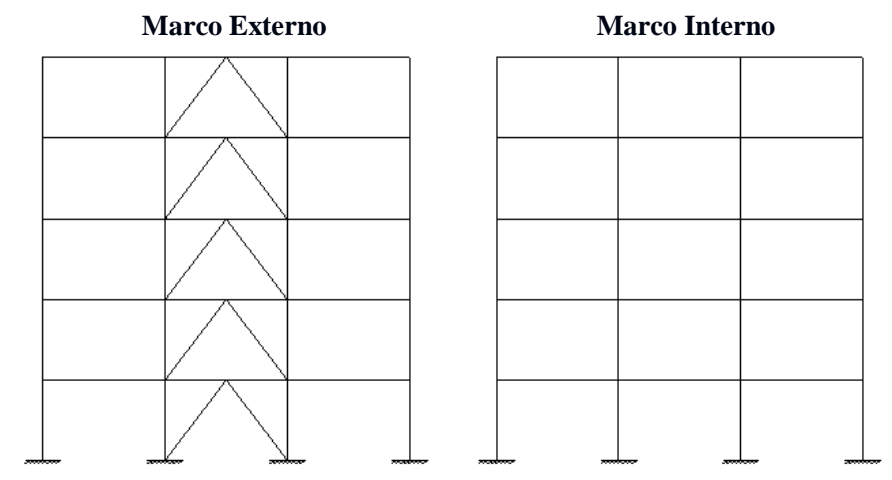

Figura 10. Sistema de contravientos desadheridos.

La Figura 11 resume la determinación del periodo fundamental de vibración requerido para controlar el desplazamiento de la estructura para los estados límite de servicio y seguridad. Los umbrales de desplazamiento se estiman conforme a la Ecuación 4 (dado que el edificio es regular y tiene pocos niveles, se han considerado, dentro de los rangos ofrecidos en la Tabla 1, los valores mínimos de $C O D$ para cada estado límite):

$$
\begin{aligned}
& \frac{\delta_{S E R}}{\alpha_{S E R}}=\frac{I D I_{S E R} H}{\alpha_{S E R} C O D_{S E R}}=\frac{0.002 \times 2000}{1.4 \times 1.2}=2.4 \mathrm{~cm} \\
& \frac{\delta_{S E G}}{\alpha_{S E G}}=\frac{I D I_{S E G} H}{\alpha_{S E G} C O D_{S E G}}=\frac{0.008 \times 2000}{1.2 \times 1.5}=8.9 \mathrm{~cm}
\end{aligned}
$$

El periodo para el cual debe diseñarse el sistema de contravientos corresponde al menor de los dos arrojados por la Figura 11, lo que resulta en $T_{M A X}=0.66 \mathrm{seg}$.

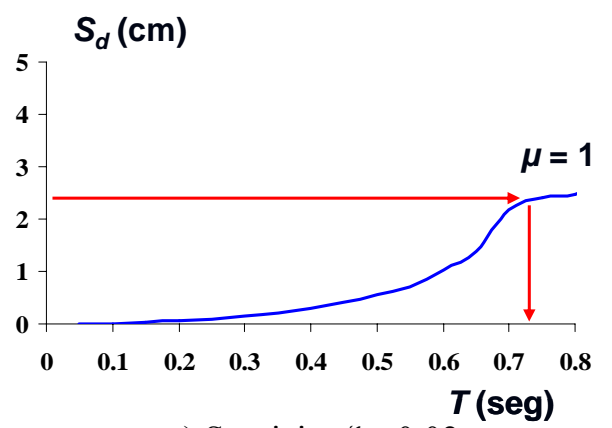

a) Servicio, $\xi=0.02$

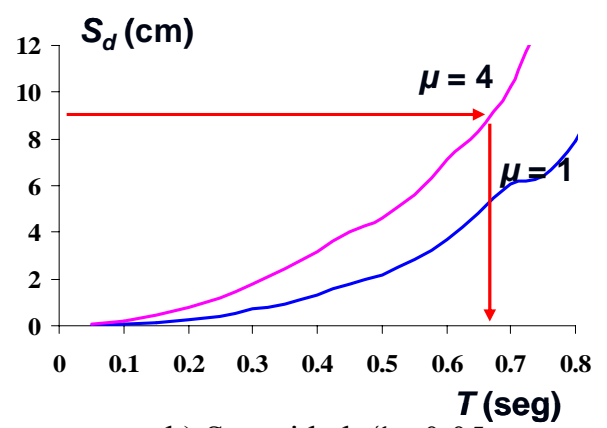

b) Seguridad, $\xi=0.05$

Figura 11. Periodos máximos para estados límite bajo consideración. 
El dimensionado por rigidez del sistema de contravientos debe hacerse de tal manera que el periodo fundamental de vibración de la estructura sea igual o ligeramente menor que $T_{M A X}$. Una posibilidad para el dimensionado consiste en establecer un modelo de análisis del edificio con contravientos, e iterar con el área de contravientos hasta obtener el periodo deseado. Existen otras posibilidades para el dimensionado, tal como se ilustra a continuación bajo la consideración de que el edificio de cinco pisos no exhibe efectos torsionales de importancia debido a su regularidad en planta (la metodología puede adaptarse con relativa facilidad al caso en que exista torsión). Primero, se utiliza el método estático para estimar las fuerzas laterales de diseño:

$$
F_{i}=c W \frac{w_{i} h_{i}}{\sum_{j=1}^{n} w_{j} h_{j}}
$$

donde $F_{i}$ es la fuerza lateral de diseño del entrepiso $i ; c$ el coeficiente sísmico de diseño; $W$ el peso de la edificación; $n$ el número de pisos; y $w_{i}$ y $h_{i}$ son el peso y altura respecto a la base, respectivamente, de la losa del piso $i$. La Tabla 6 resume las fuerzas laterales, y los correspondientes cortantes de entrepiso $\left(V_{i}\right)$ estimados conforme a la Ecuación 13 para la edificación de cinco pisos. La distribución de rigidez lateral más eficiente para dicha edificación corresponde a aquella cuya variación en altura es proporcional a la variación en altura del cortante de entrepiso. Dicha distribución se denota $K_{\text {iteórica }}$ en la Tabla 6. Conforme muestra la misma tabla, la distribución real de rigideces $\left(K_{\text {ipráctica }}\right)$ por lo general no coincide con $K_{\text {iteórica }}$, ya que debe tomar en cuenta varias consideraciones prácticas. Al respecto, suele ser práctica común en los despachos de cálculo uniformizar las secciones de los elementos estructurales en varios pisos, y tratar de evitar cortes bruscos de las propiedades estructurales en altura. Aunque en opinión de los autores la distribución $K_{\text {ipráctica }}$ presentada en la Tabla 6 representa una distribución "realista" de rigideces conforme a la práctica profesional, lo cierto es que lo más conveniente desde un punto de vista estructural es que la distribución práctica se apegue lo más posible a la teórica.

Tabla 6. Distribución de rigidez lateral en altura para el edificio de cinco pisos

\begin{tabular}{ccccc}
\hline Piso & $F_{i}$ & $V_{i}$ & $K_{\text {iteórica }}$ & $K_{\text {ipráctica }}$ \\
\hline 5 & $0.258 \mathrm{cW}$ & $0.258 \mathrm{cW}$ & $0.258 \mathrm{~K}$ & $0.7 \mathrm{~K}$ \\
4 & $0.297 \mathrm{cW}$ & $0.555 \mathrm{cW}$ & $0.555 \mathrm{~K}$ & $0.7 \mathrm{~K}$ \\
3 & $0.223 \mathrm{cW}$ & $0.778 \mathrm{cW}$ & $0.778 \mathrm{~K}$ & $1.0 \mathrm{~K}$ \\
2 & $0.148 \mathrm{cW}$ & $0.926 \mathrm{cW}$ & $0.926 \mathrm{~K}$ & $1.0 \mathrm{~K}$ \\
1 & $0.074 \mathrm{cW}$ & $1.000 \mathrm{cW}$ & $1.000 \mathrm{~K}$ & $1.0 \mathrm{~K}$ \\
\hline
\end{tabular}

Una vez que se ha determinado en términos relativos la distribución de rigidez lateral en altura, se procede a plantear las matrices de masas y de rigideces en la dirección de análisis (note que la obtención de la matriz de rigideces se basa en considerar que los entrepisos trabajan exclusivamente a corte, suposición que es razonable para edificios de pocos niveles):

$\vec{M}=\left[\begin{array}{ccccc}187.27 & 0 & 0 & 0 & 0 \\ 0 & 187.27 & 0 & 0 & 0 \\ 0 & 0 & 187.27 & 0 & 0 \\ 0 & 0 & 0 & 187.27 & 0 \\ 0 & 0 & 0 & 0 & 130.22\end{array}\right] \frac{\mathrm{kg}-\mathrm{seg}^{2}}{\mathrm{~cm}}$ 


$$
\vec{K}=\left[\begin{array}{ccccc}
2 K & -1 K & 0 & 0 & 0 \\
-1 K & 2 K & -1 K & 0 & 0 \\
0 & -1 K & 1.7 K & -0.7 K & 0 \\
0 & 0 & -0.7 K & 1.4 K & -0.7 K \\
0 & 0 & 0 & -0.7 K & 0.7 K
\end{array}\right]
$$

El proceso de dimensionado arranca al asignar un valor arbitrario (por ejemplo unitario) al parámetro $K$ indicado en la Ecuación 15. Se plantea entonces un problema de valores característicos y se estima el periodo fundamental de vibración que correspondería al sistema de contravientos $\left(T_{K}\right)$ de acuerdo al valor de $K$. La rigidez lateral de diseño para el entrepiso $i\left(K_{\text {idiseño }}\right)$ queda definida por:

$$
K_{\text {idiseño }}=K_{\text {ipráctica }}\left(\frac{T_{K}}{T_{M A X}}\right)^{2}
$$

donde $K_{\text {ipráctica }}$ corresponde a la rigidez lateral del entrepiso $i$ estimada de acuerdo al valor supuesto de $K$. El área total de contravientos requerida por cuestiones de rigidez lateral $\left(A_{i}\right)$ en el entrepiso $i$ se obtiene a partir de la Ecuación 1:

$$
A_{i}=\frac{L[\gamma+\eta(1-\gamma)]}{E \cos ^{2} \theta} K_{\text {idiseño }}
$$

En el caso del edificio de cinco pisos, se contempla $\gamma=0.5$ y $\eta=0.333$ para considerar la existencia de zonas de mayor rigidez ubicadas en los extremos de los contravientos (que incluye la existencia de placas de conexión). A partir de la aplicación de las Ecuaciones 14 a 17, se obtuvo un área total de contravientos de $84 \mathrm{~cm}^{2}$ para los tres primeros pisos, y de $56 \mathrm{~cm}^{2}$ para los dos pisos superiores; lo que resultó en un periodo fundamental de vibración $\left(T_{R E A L}\right)$ de 0.66 segundos para el sistema de contravientos.

Con el valor de $T_{R E A L}$, es posible estimar el coeficiente sísmico de diseño del edificio. Este proceso se resume con ayuda de la Figura 12. El coeficiente sísmico de diseño, que corresponde al mayor de los dos indicados en la figura, resultó ser igual a 0.175 (estado límite de seguridad), lo que a su vez resulta, para un peso de 862.6 toneladas, en un cortante basal estático de diseño de $1.1 \times 0.175 \times 862.6=166$ ton (el 1.1 representa el factor de carga). El cortante basal dinámico de diseño resultó igual a 134, lo que corresponde a $81 \%$ del cortante basal estático. Los requerimientos de área por resistencia de los contravientos se revisaron a partir de un análisis dinámico espectral que utilizó el espectro de diseño para el estado límite de seguridad reducido por un factor de sobrerresistencia de 1.2 (el cortante dinámico asociado a la revisión por resistencia es entonces $134 / 1.2=112$ ton). El valor de 1.2 supone una sobrerresistencia de $10 \%$ debido al uso de un factor de carga de 1.1 , y otro $10 \%$ debido a la contribución del sistema gravitacional a las propiedades sismorresistentes de la edificación. Note que esto implica que no hay sobrerresistencia asociada al acero con que se fabrican los contravientos; esto es, que el esfuerzo de $2375 \mathrm{~kg} / \mathrm{cm}^{2}$ es el valor esperado del esfuerzo de fluencia.

Para revisar si el área de contravientos obtenida por consideraciones de rigidez lateral es suficiente para acomodar las demandas de resistencia en los mismos, se utilizó la Ecuación 3. Para la planta baja:

$V_{b}=A_{1} f_{y} \cos \theta=84(2375) \cos 53.13^{\circ}=120>112$ ton 
por lo que no se ajustó el área de contravientos por cuestiones de resistencia. Note entonces que el área de contravientos queda regida por requerimientos de rigidez.

Los elementos de los marcos que reciben al sistema de contravientos se rediseñaron con tal fin. Para ello, se utilizó el concepto de diseño por capacidad (Virto 2006). Dados los niveles de carga axial que deben tomar las columnas de soporte, se recurrió a presforzarlas. La Figura 13 muestra la sección transversal de las vigas y columnas que soportan el sistema de contravientos.

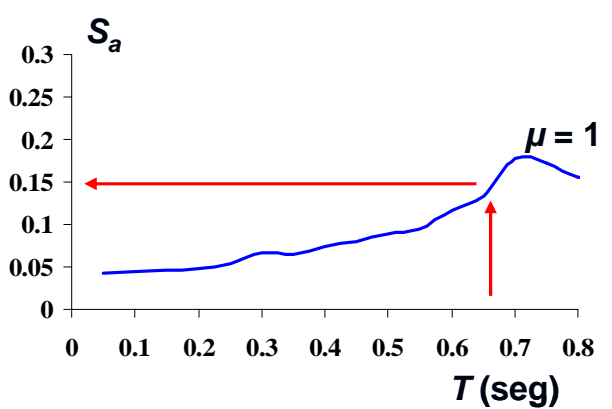

a) Servicio, $\xi=0.02$

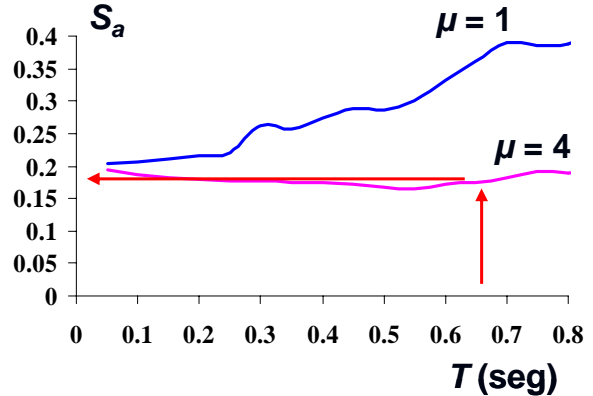

b) Seguridad, $\xi=0.05$

Figura 12. Coeficientes sísmicos de diseño para estados límite bajo consideración.
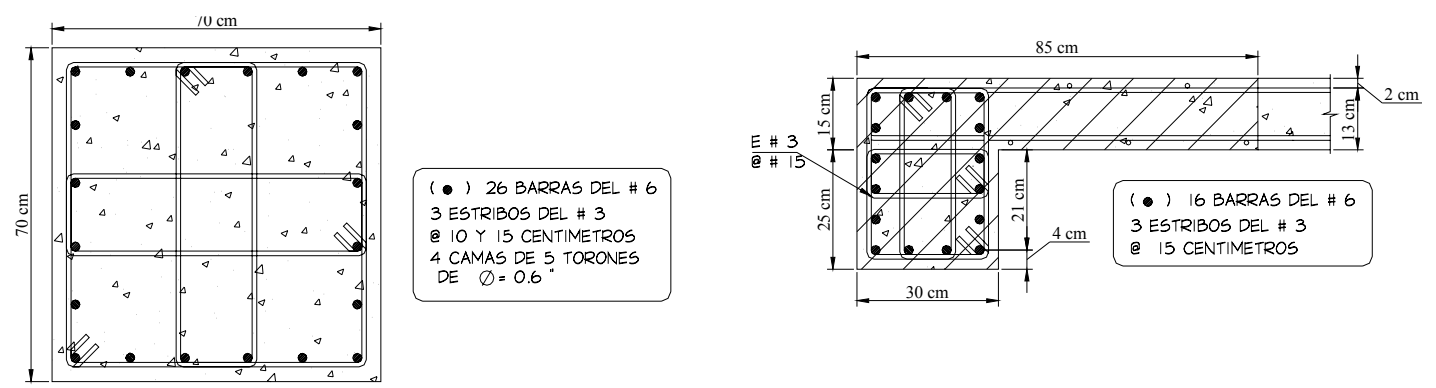

Figura 13. Sección transversal de columnas y vigas de soporte.

\section{CARACTERÍSTICAS MECÁNICAS DEL EDIFICIO CONTRAVENTEADO}

Una vez diseñado el sistema de contravientos, se procedió a establecer el modelo de análisis no lineal para el edificio contraventeado. Para ello, se añadió el sistema de contraventeo al modelo de análisis no lineal del sistema gravitacional. A los contravientos se asignó un esfuerzo de fluencia de $2375 \mathrm{~kg} / \mathrm{cm}^{2}$, lo que implica que el acero con que se fabrican no exhibe sobrerresistencia con respecto a su resistencia de diseño. La Figura 14 muestra la curva cortante basal contra desplazamiento de azotea obtenida para un patrón triangular de cargas (como referencia, la figura también incluye la curva correspondiente al sistema gravitacional).

La Figura 15, que ilustra la distribución en altura de la distorsión de entrepiso, muestra que la edificación alcanza distorsiones máximas de 0.002 y 0.008 para desplazamientos de azotea de 3.4 y 10.6 $\mathrm{cm}$, respectivamente. Note que estos valores están muy cercanos a los valores de $\delta_{S E R}$ y $\delta_{S E G}$ estimados con fines de prediseño a partir de la Ecuación $12(2.4 \times 1.4=3.4$ y $8.9 \times 1.2=10.7 \mathrm{~cm}$, respectivamente $)$. Conforme muestra la Figura 14, la idealización bilineal de la curva de desplazamiento de azotea contra 
cortante basal arroja un desplazamiento y un cortante basal idealizados de fluencia de $3.6 \mathrm{~cm}$ y 175 toneladas, respectivamente; lo que implica un nivel de sobrerresistencia del orden de $50 \%$ con relación al cortante basal dinámico utilizado para revisar por resistencia el área de los contravientos $(175 / 112 \approx 1.56)$. Puede decirse que el factor de sobrerresistencia de 1.2 utilizado durante el diseño es conservador, ya que la estructura exhibe otras fuentes de sobrerresistencia. En particular, el sistema gravitacional desarrolla un cortante basal cercano a las 35 toneladas para un desplazamiento de azotea de $3.6 \mathrm{~cm}$, lo que aunado al cortante basal de 120 toneladas que desarrolla el sistema de contravientos (Ecuación 18), resulta en un cortante basal total de 155 toneladas (valor cercano al indicado en la Figura 14 para un desplazamiento de azotea de $3.6 \mathrm{~cm}$ ).

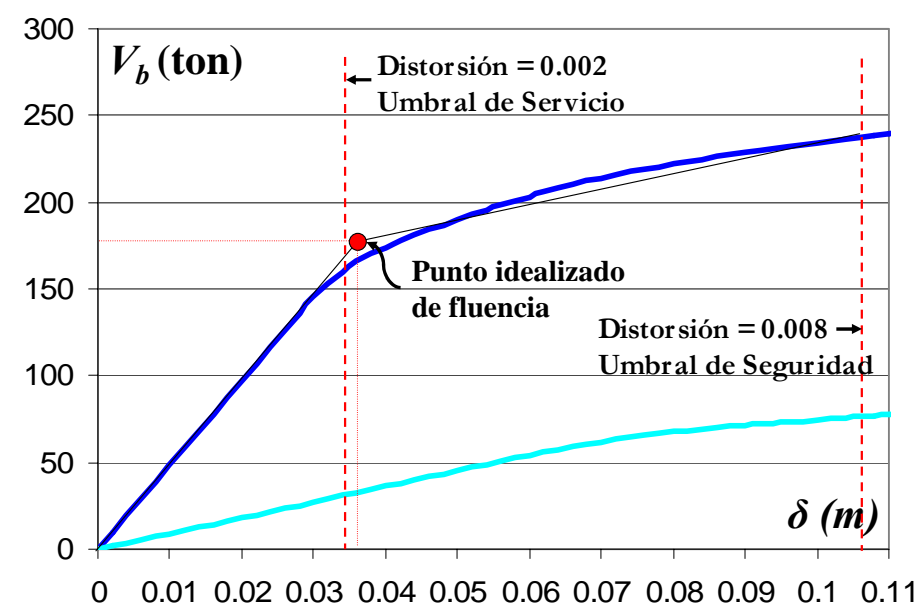

Figura 14. Curva de desplazamiento de azotea contra cortante basal de edificio contraventeado.

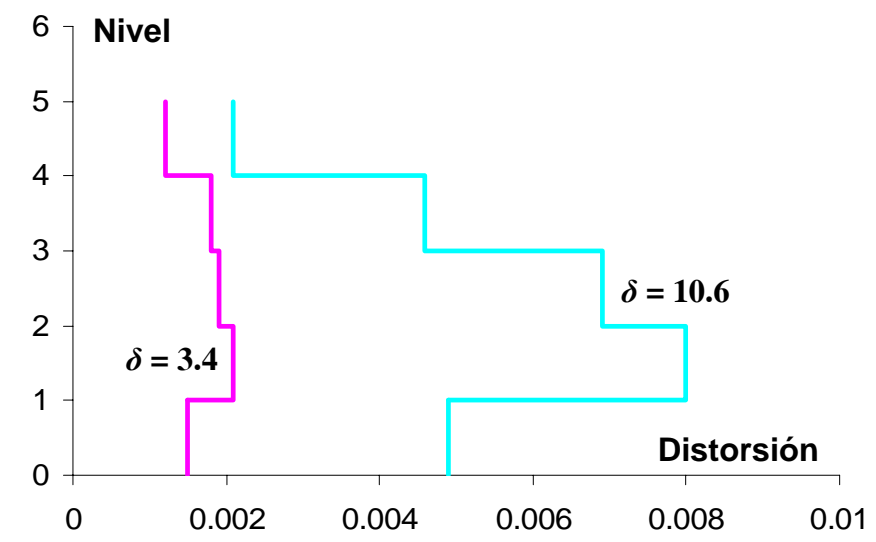

Figura 15. Distorsiones de entrepiso en función del desplazamiento de azotea, edificio contraventeado.

La Tabla 7 resume en su segunda columna las propiedades estructurales globales del edificio contraventeado, y las compara con sus valores de diseño. Debido a que no se consideró la contribución del sistema gravitacional durante el diseño del sistema de contravientos, las propiedades estructurales para el edificio contraventeado implican mayor rigidez y resistencia lateral que las requeridas de acuerdo a la metodología de diseño. 
Tabla 7. Propiedades estructurales de edificio contraventeado de cinco pisos

\begin{tabular}{cccc}
\hline Propiedad Estructural & Valor de Diseño & Versión Original & Segunda Versión \\
\hline $\begin{array}{c}\text { Periodo Fundamental } \\
(\mathrm{seg})\end{array}$ & 0.66 & 0.62 & 0.68 \\
\hline $\begin{array}{c}\text { Cortante Basal de } \\
\text { Fluencia (ton) }\end{array}$ & 134 & 175 & 145 \\
\hline
\end{tabular}

\section{DESEMPEÑO SÍSMICO DEL EDIFICIO CONTRAVENTEADO}

Para establecer el desempeño sísmico del edificio contraventeado, se sujetó al modelo de análisis no lineal a los acelerogramas considerados en las Tablas 4 y 5 . La Tabla 8 resume las demandas máximas de desplazamiento de azotea $(\delta)$ y distorsión $(D I)$ para cada estado límite bajo consideración, y reporta su media, desviación estándar y la media más una desviación estándar. Además, para el estado límite de seguridad, la Tabla 8 incluye las demandas máximas de rotación plástica en la viga y columna críticas $\left(\theta_{\text {pviga }}\right.$ y $\theta_{\text {pcolumna }}$, respectivamente). El modelo de análisis no lineal del edificio considero un porcentaje de amortiguamiento crítico de $2 \%$ para el estado límite de servicio, y de $5 \%$ para el estado límite de seguridad. Se utilizó amortiguamiento viscoso a través de una matriz de amortiguamiento de Rayleigh, asignando el porcentaje de amortiguamiento crítico de interés a los dos primeros modos del edificio.

Tabla 8. Demandas sísmicas correspondientes a los estados límite de servicio y seguridad

\begin{tabular}{|c|c|c|c|c|c|c|c|}
\hline \multicolumn{3}{|c|}{ Estado Límite de Servicio } & \multicolumn{5}{|c|}{ Estado Límite de Seguridad } \\
\hline Acelerograma & $\delta(\mathrm{cm})$ & $D I$ & Acelerograma & $\delta(\mathrm{cm})$ & $D I$ & $\theta_{\text {pviga }}$ & $\theta_{\text {pcolumna }}$ \\
\hline s31 & 1.73 & .0011 & $m x 01$ & 4.44 & .0036 & .0000 & .0005 \\
\hline s32 & 2.21 & .0014 & $m x 03$ & 3.39 & .0024 & .0000 & .0000 \\
\hline$s 43$ & 1.55 & .0010 & $m x 04$ & 3.92 & .0031 & .0000 & .0002 \\
\hline$s 45$ & 1.14 & .0007 & $m x 06$ & 4.37 & .0036 & .0000 & .0006 \\
\hline$s 46$ & 1.60 & .0010 & $m x 07$ & 6.07 & .0051 & .0019 & .0016 \\
\hline$s 51$ & 1.11 & .0007 & $\operatorname{mx} 08$ & 3.77 & .0028 & .0000 & .0000 \\
\hline$s 59$ & 0.48 & .0003 & & & & & \\
\hline$s 77$ & 0.99 & .0006 & & & & & \\
\hline$s 79$ & 1.25 & .0008 & & & & & \\
\hline$s 89$ & 1.65 & .0010 & & & & & \\
\hline$s 90$ & 2.22 & .0014 & & & & & \\
\hline s119 & 1.48 & .0009 & & & & & \\
\hline$s 125$ & 1.51 & .0010 & & & & & \\
\hline s129 & 0.61 & .0004 & & & & & \\
\hline s131 & 1.04 & .0007 & & & & & \\
\hline s143 & 0.75 & .0005 & & & & & \\
\hline s144 & 1.03 & .0007 & & & & & \\
\hline Media & 1.31 & .0008 & Media & 4.33 & .0034 & .0003 & .0005 \\
\hline$\sigma$ & 0.50 & .0003 & $\sigma$ & 0.94 & .0009 & .0008 & .0006 \\
\hline Media $+\sigma$ & 1.81 & .0012 & Media $+\sigma$ & 5.27 & .0044 & .0011 & .0011 \\
\hline
\end{tabular}

La Figura 16 contrapone los resultados resumidos en la Tabla 8 (media $+\sigma$ ) a la curva desplazamiento de azotea contra cortante basal del edificio. Mientras que el círculo blanco representa las demandas máximas esperadas para el estado límite de servicio, el círculo gris corresponde al estado límite de seguridad. Las líneas verticales discontinuas indican los umbrales de desplazamiento de azotea que 
corresponden a los umbrales de distorsión aceptables para ambos estados límite. Se observa que los contravientos no fluyen para el estado límite de servicio, y que la demanda de desplazamiento de azotea correspondiente al estado límite de seguridad es considerablemente menor que el umbral de $10.6 \mathrm{~cm}$ considerado como aceptable. En la evaluación de los resultados mostrados, es importante hacer notar que existe un nivel de sobrediseño importante en la estructura.

Para estudiar el desempeño sísmico de la edificación para un estado cercano al umbral de seguridad, se decidió sujetar al modelo no lineal del edificio contraventeado a los sismos de la Tabla 5 escalados por un factor de 1.4. Mientras que el círculo negro en la Figura 16 corresponde al desplazamiento medio $+\sigma$ de azotea de esta nueva serie de análisis, la Tabla 9 aporta detalles de las demandas de deformación en el edificio.

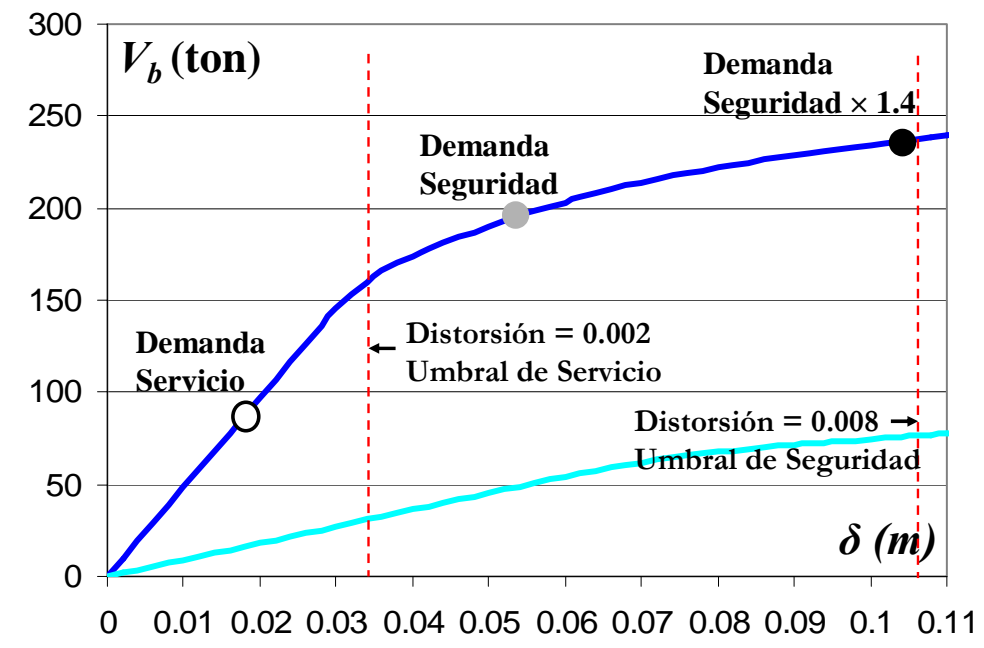

Figura 16. Desempeño estructural de la primera versión del edificio contraventeado.

Tabla 9. Demandas sísmicas correspondientes a acelerogramas de la

Tabla 4 escalados por 1.4

\begin{tabular}{ccccc}
\hline Acelerograma & $\delta(\mathrm{cm})$ & $D I$ & $\theta_{\text {pviga }}$ & $\theta_{\text {pcolumna }}$ \\
\hline$m x 01$ & 8.09 & .0069 & .0040 & .0025 \\
$m x 03$ & 4.62 & .0036 & .0000 & .0006 \\
$m x 04$ & 7.56 & .0062 & .0020 & .0021 \\
$m x 06$ & 10.85 & .0092 & .0065 & .0038 \\
$m x 07$ & 10.46 & .0090 & .0066 & .0040 \\
$m x 08$ & 5.19 & .0043 & .0004 & .0009 \\
\hline Media & 7.80 & .0065 & .0033 & .0023 \\
\hline$\sigma$ & 2.59 & .0023 & .0029 & .0014 \\
\hline Media $+\sigma$ & 10.38 & .0089 & .0061 & .0038 \\
\hline
\end{tabular}

A partir de la comparación de los resultados arrojados por la Tabla 9 (demandas de distorsión y rotación plástica de 0.0089 y 0.0061 , respectivamente, para un desplazamiento de azotea de $10.4 \mathrm{~cm}$ ) con las condiciones de diseño para el estado límite de seguridad (umbrales de distorsión y rotación plástica de 0.0080 y 0.0050 , respectivamente, para un desplazamiento de azotea de $10.6 \mathrm{~cm}$ ), puede decirse que la 
metodología propuesta resulta en un comportamiento razonable de la estructura cuando esta se acerca al umbral de seguridad.

\section{OBSERVACIONES FINALES}

La aplicación de la metodología propuesta puede considerarse exitosa a pesar de haber dado lugar a un diseño ligeramente conservador. Al respecto, lo conservador de su aplicación se debe a los mayores niveles de rigidez y resistencia lateral que resultan por no considerar durante el diseño la contribución del sistema gravitacional. De considerarlo deseable, el diseñador puede tomar en cuenta la contribución del sistema gravitacional, e incluso diseñarlo para que acomode un porcentaje mayor de la carga lateral. Una opción más es que se utilice el sistema de contraventeo para reforzar una estructura existente.

La metodología presentada aquí puede adaptarse fácilmente para tomar en cuenta la contribución del sistema gravitacional o de un sistema estructural existente. Para ello, es necesario llevar a cabo, tal como indica la metodología propuesta, un análisis estático no lineal para establecer el periodo fundamental de vibración $\left(T_{G V}\right)$ y la curva cortante basal contra desplazamiento de azotea del sistema gravitacional o existente. Bajo la consideración de que la respuesta lateral de la edificación está dominada por efectos globales de corte, es posible establecer que los sistemas de contraventeo y gravitacional o existente trabajan como dos sistemas en paralelo, de tal manera que la rigidez lateral de la edificación puede estimarse como la suma de las rigideces laterales de ambos sistemas. Bajo este supuesto, puede plantearse lo siguiente:

$\frac{1}{T_{C V}^{2}}+\frac{1}{T_{G V}^{2}}=\frac{1}{T_{M A X}^{2}} \Rightarrow \frac{1}{T_{C V}^{2}}=\frac{1}{T_{M A X}^{2}}-\frac{1}{T_{G V}^{2}}$

donde $T_{M A X}$ sigue indicando el periodo para el cual debe diseñarse la edificación, y $T_{C V}$ indica el periodo para el cual deben dimensionarse los contravientos. Note que según la Ecuación 19, el valor de $T_{C V}$ resulta mayor que el de $T_{M A X}$, lo que indica que debido a la contribución del sistema gravitacional, los requerimientos de rigidez en los contravientos se reducen. En el caso del edificio de 5 pisos, la Ecuación 19 resulta en:

$\frac{1}{T_{C V}^{2}}=\frac{1}{0.66^{2}}-\frac{1}{1.44^{2}} \Rightarrow T_{C V}=0.74$ segundos

Aplicando las Ecuaciones 13 a 17 para un periodo de 0.74 segundos (en lugar del valor de 0.66 considerado antes), se obtienen áreas de contravientos que son $20 \%$ menores que las manejadas para la versión original del edificio contraventeado. Esto resulta en áreas de 67.2 y $44.8 \mathrm{~cm}^{2}$ para los primeros tres pisos y los dos pisos superiores, respectivamente.

La contribución del sistema gravitacional o existente también puede tomarse en cuenta durante la revisión del área de contravientos por cuestiones de resistencia. En este caso, se resta al cortante basal de diseño $\left(V_{b d}\right)$ el cortante basal que desarrolla el sistema gravitacional $\left(V_{G V}\right)$ para el desplazamiento de fluencia del edificio contraventeado. Para el caso del edificio de cinco pisos, la Ecuación 12a arroja un desplazamiento de fluencia igual a $3.4 \mathrm{~cm}$, al que corresponde, según la Figura 8 , un cortante basal $V_{G V}$ de 31 toneladas. Bajo consideración de un factor de sobrerresistencia $(S R)$, que en este caso es igual a 1.1, se obtiene el cortante basal de diseño para el sistema de contravientos $\left(V_{C V}\right)$ : 


$$
V_{C V}=\frac{V_{b d}-V_{G V}}{S R}=\frac{134-31}{1.1}=94 \text { ton }
$$

Note que en este caso, el factor $S R$ no contempla la posible contribución del sistema gravitacional, ya que esta ha sido tomada en cuenta explícitamente durante el proceso de diseño. La aplicación de la Ecuación 3 para la segunda versión del sistema de contraventeo resulta en:

$V_{b}=A_{1} f_{y} \cos \theta=67.2(2375) \cos 53.13^{\circ}=96$ ton $>94$ ton

de tal manera que las áreas de contravientos no requieren ajustarse por cuestiones de resistencia.

Mientras que la Figura 17 muestra la curva cortante basal contra desplazamiento de azotea para la segunda versión del edificio contraventeado, la Tabla 7 resume en su tercera columna las propiedades estructurales globales del edificio, y las compara con sus valores de diseño. Note que en el caso de la segunda versión del edificio, las propiedades estructurales globales que se estiman se encuentran muy cercanas a sus respectivos valores de diseño. La Figura 17 incluye además las demandas de desplazamiento de azotea para los dos estados límite bajo consideración, y las compara con sus respectivos umbrales de diseño. Se obtuvieron demandas media $+\sigma$ de desplazamiento de azotea de 2.64 y 8.24 centímetros para los estados límite de servicio y seguridad, respectivamente. A estas corresponden demandas de distorsión de 0.0017 y 0.0067 , respectivamente, que se encuentran razonablemente cercanas a los umbrales de diseño de 0.002 y 0.008 , respectivamente.

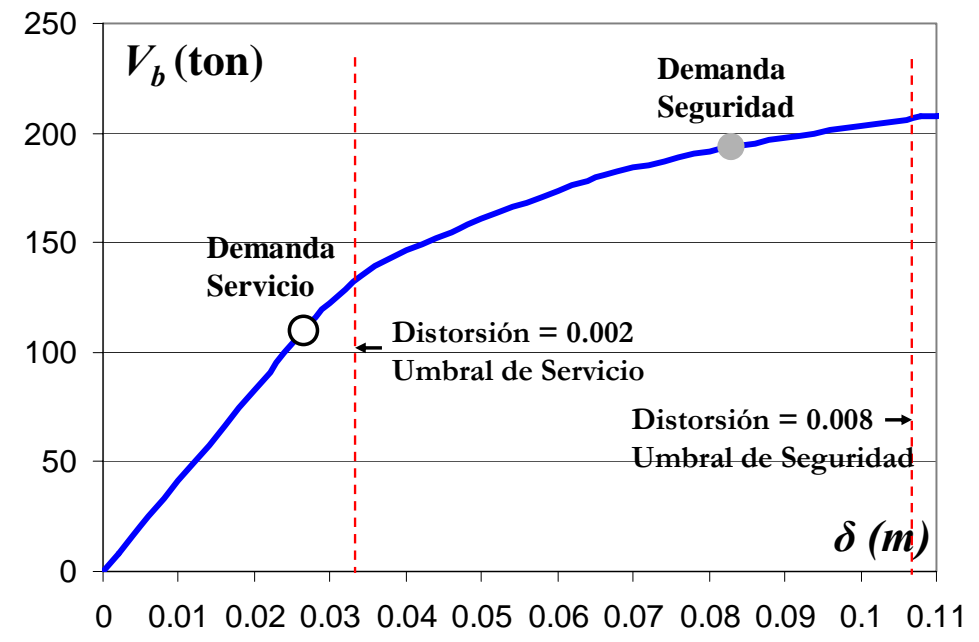

Figura 17. Desempeño estructural de la segunda versión del edificio contraventeado.

Otra consideración que los autores quisieran resaltar es el hecho que durante el diseño de la estructura se utilizó una ductilidad máxima de cuatro para definir los espectros de diseño correspondientes al estado límite de seguridad. Sin embargo, conforme puede concluirse a partir de la Figura 14, el edificio de cinco pisos desarrolla para el umbral de deformación de seguridad una ductilidad máxima de $10.6 / 3.6 \approx 3$. Al respecto, la ductilidad global resultó relativamente baja respecto al valor inicialmente contemplado porque existe una concentración significativa de deformación plástica en los entrepisos 2 y 3 del edificio. Una ductilidad global de cuatro hubiera requerido de una distribución más uniforme del comportamiento plástico en los diferentes entrepisos, lo que a su vez hubiera implicado establecer una distribución $K_{\text {ipráctica }}$ que coincidiera mejor con la distribución $K_{\text {iteórica }}$ resumida en la Tabla 6 . Con base en 
lo anterior los autores consideran que es conveniente variar el tamaño de los contravientos en todos los entrepisos de una edificación de acuerdo a la distribución de cortante de entrepiso.

La distribución y ubicación de los contravientos son relevantes para la seguridad estructural de la edificación y pueden llegar a afectar de manera importante el diseño de los elementos de soporte. En el ejemplo ilustrativo se decidió concentrar el sistema de contraventeo en las crujías centrales de los marcos exteriores, y se consideró conveniente asignarle un alto porcentaje de la carga lateral de diseño. Debido a que las dos crujías contraventeadas bajan un elevado porcentaje del cortante basal y momento de volteo que corresponden al edificio completo, el edificio exhibe una baja redundancia y fue necesario presforzar las columnas que soportan los contravientos. En caso de que el ingeniero estructural considere esto como inconveniente, puede incrementar la redundancia del sistema y reducir las acciones sobre los elementos de soporte a través de: A) Incrementar la rigidez lateral del sistema gravitacional, ó B) Contraventear más crujías. Note que en términos generales, la opción A implica que el daño estructural en la estructura compuesta no se concentra en los contravientos, sino que se extiende a vigas y columnas, y que es necesario considerar explícitamente durante el proceso de diseño la contribución del sistema gravitacional a las propiedades sismorresistentes de la estructura.

Finalmente, es necesario mencionar políticas para la sustitución de contravientos dañados. Como se comentó con anterioridad, reparar el daño estructural de una edificación diseñada conforme a los supuestos de este artículo consiste en sustituir los contravientos que fluyeron en demasía durante una excitación sísmica severa. Un desplazamiento residual (permanente) importante en la edificación es un indicativo de niveles altos de fluencia en el sistema de contraventeo. Por tanto, será necesario establecer, después de una excitación sísmica severa, si los diferentes entrepisos de la edificación exhiben un desplome importante. En caso afirmativo, será necesario reemplazar los contravientos de los entrepisos que exhiban dicho desplome.

\section{CONCLUSIONES}

Dentro del contexto de una metodología de diseño sísmico basada en el control explícito del desplazamiento lateral, el área de contravientos requerida por rigidez lateral debe determinarse en función del periodo fundamental requerido por la estructura para controlar el nivel de daño en los sistemas gravitacional y no estructural. El área de contravientos requerida por resistencia debe ser tal que impida la fluencia excesiva del sistema de contravientos durante la excitación sísmica de servicio, y que dé lugar a una disipación de energía adecuada durante la excitación sísmica de seguridad.

La aplicación de la metodología propuesta a una edificación de cinco pisos ha dado lugar a un nivel de diseño razonablemente conservador para los estados límite de servicio y seguridad. Además, el comportamiento local que la edificación exhibe cuando su desplazamiento de azotea se acerca al umbral de seguridad de vida es congruente con el planteamiento de diseño. Los marcos gravitacionales del ejemplo ilustrativo resultan muy ligeros con respecto a los que tendría una edificación similar diseñada para resistir simultáneamente cargas verticales y sísmicas. No solo eso, sino que los tamaños de vigas y columnas de los marcos gravitacionales, así como su detallado mínimo, es uniforme a través de todo el edificio. Esto implica grandes ahorros en cuanto a costos de materiales y construcción. La cantidad de acero concentrada en los contravientos representa un importante ahorro de este material, además de que el daño estructural que pudiera exhibir la edificación se repara sustituyendo exclusivamente los contravientos que hayan fluido durante la excitación sísmica. 
La distribución y ubicación de los contravientos es relevante para la seguridad estructural de la edificación. En el ejemplo ilustrativo se decidió concentrar el sistema de contraventeo en las crujías centrales de los marcos exteriores. El problema con este tipo de arreglo es que existe poca redundancia, principalmente por la poca cantidad de plastificaciones requeridas para que se forme el mecanismo plástico del sistema de contraventeo.

A continuación se presentan algunos puntos considerados como importantes para investigaciones futuras concernientes al uso de contravientos desadheridos en México: A) Conexiones.- Es importante estudiar el efecto de la concentración de esfuerzos en la zona de conexión, y establecer el detallado requerido para que el sistema de contravientos acomode de manera estable dichos esfuerzos; B) Aplicación a edificios de mayor altura.- En este trabajo se despreciaron los efectos de deformación por flexión global del sistema de contravientos, los cuales son relevantes para la respuesta sísmica de edificios altos y esbeltos; y C) Costos.- Es importante comparar los costos inicial y total de edificaciones similares resueltas con un sistema de contravientos desadheridos y con sistemas estructurales tradicionales.

\section{RECONOCIMIENTOS}

Los autores agradecen el apoyo de la Universidad Autónoma Metropolitana.

\section{REFERENCIAS}

Applied Technology Council (1998), "FEMA 306. Evaluation of earthquake damaged concrete and masonry wall buildings", ATC-43 Project.

Arroyo, D y M Ordaz (2007), "Hysteretic Energy Demands for SDOF Systems Subjected to Narrow Band Earthquake Ground Motions. Applications to the Lake Bed Zone of Mexico City", Journal of Earthquake Engineering, 11, 147-165.

Badillo, A H (2000) "Criterio de diseño sísmico por desempeño para reforzar edificios con disipadores de energía” Tesis de Maestría, Universidad Nacional Autónoma de México.

Bentz, E y M P Collins (2000), “Response 2000”, http://www.ecf.utoronto.ca/ bentz.

Bertero, V V, Anderson, J C , Krawinkler, H y E Miranda (1991), "Design guidelines for ductility and drift limits: review of state-of-the-practice and state-of-the-art in ductility and drift-based earthquakeresistant design of buildings", Reporte UCB/EERC-91/15, Universidad de California en Berkeley.

Bertero, V V (1997), "Performance-based seismic engineering: A critical review of proposed guidelines", Seismic Design Methodologies for the Next Generation of Codes, Slovenia, Memorias, 1-31.

Black, C, Makris, N y I Aiken (2002), "Component Testing, stability analysis and characterization of buckling-restrained unbounded braces", Reporte PEER 2002/08, Universidad de California en Berkeley.

Bojórquez, E y S E Ruiz (2004) "Strength reduction factors for the valley of Mexico taking into account low cycle fatigue effects", $13^{\text {th }}$ World Conference on Earthquake Engineering, Memorias, Artículo 516.

Chen, C H, Hsiao, P C, Lai, J W, Lin, M L, Weng, Y T y K C Tsai (2004), "Pseudo-dynamic test of a full-scale CFT/BRB frame: Part 2 - Construction and testing", $13^{\text {th }}$ World Conference on Earthquake Engineering, Memorias, Artículo 2175.

Chopra, A K (2001), "Dynamics of structures, Theory and applications to earthquake engineering", Editorial Prentice Hall, Segunda Edición. 
Clark, P, Kasai, K, Aiken, I y I Kimura (2000), "Evaluation of Design Methodologies for Structures Incorporating Steel Unbonded Braces for Energy Dissipation," $12^{\text {th }}$ World Conference on Earthquake Engineering, Memorias, Artículo 2240.

Federal Emergency Management Agency (2000), "FEMA 356, Prestandard and commentary for the seismic rehabilitation of buildings".

Huerta-Garnica, B y E Reinoso-Angulo (2002), "Espectros de energía de movimientos fuertes registrados en México", Revista de Ingeniería Sísmica, 66, 45-72.

Ko, E, Mole, A, Aiken, I, Tajirian, F, Rubel, Z y I Kimura (2002), "Application of the unbonded brace in medical facilities", $7^{\text {th }}$ National Conference on Earthquake Engineering, Memorias, Artículo 514.

Lopez, W, Gwie, D, Saunders, M y T Lauck (2002), "Lessons learned from large-scale tests of unbonded braced frame subassemblage", SEAOC 71st Annual Convention, Memorias.

Mahin, S, Uriz, P, Aiken, I, Field, C y E Ko (2004), "Seismic performance of buckling restrained braced frame systems", $13^{\text {th }}$ World Conference on Earthquake Engineering, Memorias, Artículo 1681.

Panagiotakos, T B y M N Fardis (2001), "Deformations of reinforced concrete members at yielding and ultimate", ACI Structural Journal, 98(2), 135-148.

Pantazopoulou, S J y C W French (2001), "Slab participation in practical earthquake design of reinforced concrete frames", ACI Structural Journal, 98 (4), 479-489.

Prakash, V, Powell, G H y S Campbell (1993), "DRAIN-2DX Base program description and user guide", Reporte UCB/SEMM-93/17, Universidad de California en Berkeley.

Qi, X y J P Moehle (1991), "Displacement design approach for reinforced concrete structures subjected to earthquakes", Reporte No. UCB/EERC-91/02, Universidad de California en Berkeley.

Reyes Salinas, C (1999), "El estado límite de servicio en el diseño sísmico de edificios", Tesis de Doctorado, Universidad Nacional Autónoma de México.

Rodriguez, M E y J C Aristizabal (1999), "Evaluation of a seismic damage parameter", Earthquake Engineering and Structural Dynamics, 28, 463-477.

Sánchez-Badillo, A y A Terán-Gilmore (2008), "Diseño por desempeño de estructuras dúctiles de concreto reforzado ubicadas en la Zona del Lago del D.F.: ejemplo de aplicación”, Revista de Ingeniería Sísmica, artículo aceptado para publicación.

Terán-Gilmore, A (1998), "Características mecánicas y desempeño sísmico de marcos dúctiles de concreto reforzado", XI Congreso Nacional de Ingeniería Estructural, I, 564-573.

Terán-Gilmore, A (2004), "On the use of spectra to establish damage control in regular frames during global predesign”, Earthquake Spectra, 20(3), 1-26.

Terán-Gilmore, A y M Espinoza-Johnson (2008), "Diseño por desempeño de estructuras dúctiles de concreto reforzado ubicadas en la Zona del Lago del D.F.: la resistencia lateral de diseño", Revista de Ingeniería Sísmica, artículo aceptado para publicación.

Terán-Gilmore, A y R Simón (2008), "Use of cumulative ductility spectra within a deformation-control format for seismic design of ductile structures subjected to long duration motions", Journal of Earthquake Engineering, 12, 136-151.

Terán-Gilmore, A y N Bahena-Arredondo (2008), "Cumulative ductility spectra for seismic design of ductile structures subjected to long duration motions: concept and theoretical background", Journal of Earthquake Engineering, 12, 152-172. 
Tremblay, R, Degrange, G y J Blouin (1999), "Seismic rehabilitation of a four-story building with a stiffened bracing system", $8^{\text {th }}$ Canadian Conference on Earthquake Engineering, Memorias.

Tremblay, R, Bolduc, P, Neville, R y R DeVall (2006), "Seismic testing and performance of bucklingrestrained bracing systems", Canadian Journal of Civil Engineering, 33, 183-198.

Uang, C M y M Nakashima (2003), "Steel buckling-restrained braced frames", Earthquake Engineering: Recent Advances and Applications, Capítulo 16, CRC Press.

Uang, C M y S Kiggins (2003), "Reducing residual drift of buckling-restrained braced frames as a dual system", International Workshop on Steel and Concrete Composite Construction (IWSCCC-2003), Reporte No. NCREE-03-026, 189-198.

Virto, N (2006), "Sistema Pasivo de disipación de energía para edificios de mediana altura desplantados e la Zona del Lago del D.F.", Tesis de Maestría, Universidad Autónoma Metropolitana.

Watanabe, A, Hitomi, Y, Saeki, E, Wada, A y M Fujimoto (1988), "Properties of Brace Encased in Buckling-Restraining Concrete and Steel Tube." $9^{\text {th }}$ World Conference on Earthquake Engineering, Memorias, IV, 719-724. 